\title{
An Empirical Model for Dinitrogen Gas Emission from Inland Waters
}

\author{
Elizabeth Sikar ${ }^{1}$, Marco Aurelio dos Santos ${ }^{2}$, Ednaldo Oliveira dos Santos ${ }^{3}$ \\ ${ }^{1}$ Rua Sebastião de Moraes, São Carlos, Brazil \\ ${ }^{2}$ Energy Planning Program/COPPE/UFRJ, Centro de Tecnologia, Bloco C, Sala 211, Cidade Universitária, \\ Rio de Janeiro, Brazil \\ ${ }^{3}$ Department of Environmental Sciences, Federal Rural University of Rio de Janeiro, \\ Seropédica, Brazil \\ Email: *emsikar@gmail.com
}

How to cite this paper: Sikar, E., dos Santos, M.A. and dos Santos, E.O. (2019) An Empirical Model for Dinitrogen Gas Emission from Inland Waters. Atmospheric and Climate Sciences, 9, 1-25.

https://doi.org/10.4236/acs.2019.91001

Received: October 16, 2018

Accepted: November 30, 2018

Published: December 3, 2018

Copyright (C) 2019 by authors and Scientific Research Publishing Inc. This work is licensed under the Creative Commons Attribution International License (CC BY 4.0)

http://creativecommons.org/licenses/by/4.0/

(c) (i) Open Access

\begin{abstract}
The motivation to calculate this empirical model resulted from often observing-at the time disconcerting-excess dinitrogen gas $\left(\mathrm{N}_{2}\right.$ concentration $>$ background concentration) in bubble-gas emission samples, collected primarily for the purpose of carbon budget research, from Brazilian rivers and reservoirs sampled during roughly 100 field surveys lasting 4 days each on average and executed between years 2000 and 2012. We model the (serendipitously) measured dinitrogen gas above environmental concentration $\left(\mathrm{N}_{2} \mathrm{aec}\right)$ escaping in bubbles from Brazilian rivers as a function of dissolved nitrogen $(\mathrm{N})$ in water. To this model, we mathematically add a pre-existing model of diffusively emitted denitrified dinitrogen (also as a function of dissolved $\mathrm{N}$ ) from streams in the United States of America (USA). The resulting model predicts denitrified dinitrogen water-air emission from inland waters in the USA, China and Germany.
\end{abstract}

\section{Keywords}

Dinitrogen Gas Emission, Inland Waters, Bubbles, Diffusion, Nitrogen Cycle, Denitrification

\section{Introduction}

Gases evade water bodies through the diffusive, ebullitive and advective pathways [1] [2]. Diffusive emissions of greenhouse gases (GHG) such as carbon dioxide $\left(\mathrm{CO}_{2}\right)$, methane $\left(\mathrm{CH}_{4}\right)$, and nitrous oxide can be directly measured using the concentration increase rate of these gases in the headspace of floating static chambers. The thin boundary layer method is also used [3]. However, floating static 
chambers are not used to sample diffusive emissions of our subject dinitrogen gas above environmental concentration $\left(\mathrm{N}_{2} \mathrm{aec}\right)$ - such as denitrified $\mathrm{N}_{2}$-because of the high concentration of atmospheric $\mathrm{N}_{2}$ (78\%) in the headspace and the difficulty to distinguish $\mathrm{N}_{2}$ aec from environmental $\mathrm{N}_{2}$ [4]. As for bubble emissions of GHG, they are sampled carrying out a different procedure in which equipment similar to inverted funnels [5] [6] is used, thus allowing ebullitive emission sampling to be done independently of the diffusive one. While the difficulty to distinguish environmental $\mathrm{N}_{2}$ from $\mathrm{N}_{2}$ aec in diffusively emitted gas samples exists for bubble-gas samples too, this can be dealt with by using argon (Ar) as a tracer of environmental $\mathrm{N}_{2}$ in bubble-gas - a path here taken.

The ebullitive:diffusive water-air emission partition ratio for a certain gas can be explained in part by its solubility in water and atmospheric concentration. For instance, ebullitive water-air emission of $\mathrm{CO}_{2}$ tends to be very small. Typically, there is less than $1 \% \mathrm{CO}_{2}$ in the gas composition of bubbles escaping tropical reservoirs [7]. On the other hand, $\mathrm{CO}_{2}$ water-air emission occurs via the diffusive pathway mainly for two reasons. First, it is highly soluble in water ( 88 $\mathrm{ml} / 100 \mathrm{ml} \mathrm{H}_{2} \mathrm{O}$ at $20^{\circ} \mathrm{C}$ ) [8]. Second, water bodies tend to be under saturated with dissolved $\mathrm{CO}_{2}$ because of the small-although increasing-concentration of $\mathrm{CO}_{2}$ in Earth's atmosphere (485 ppm in year 2015) [9]. (Dissolved $\mathrm{CO}_{2}$ saturated water bodies exist under a $100 \% \mathrm{CO}_{2}$ atmosphere. Under this scenario, further generation of $\mathrm{CO}_{2}$ would increasingly super saturate water bodies with dissolved $\mathrm{CO}_{2}$. Then, concentration of $\mathrm{CO}_{2}$ in emitted bubbles would tend to increase with $\mathrm{CO}_{2}$ production, simply because streams, rivers and reservoirs could not dissolve $\mathrm{CO}_{2}$ indefinitely and therefore diffusively emitted $\mathrm{CO}_{2}$ could not increase indefinitely).

Solubility of $\mathrm{CH}_{4}\left(3.5 \mathrm{ml} / 100 \mathrm{ml} \mathrm{H}_{2} \mathrm{O}\right.$ at $\left.17^{\circ} \mathrm{C}\right)$ [8] is much smaller compared to that of $\mathrm{CO}_{2}$. Because of the small concentration of $\mathrm{CH}_{4}$ in air $(1.84 \mathrm{ppm}$ in year 2015) [9] water bodies are also under saturated with dissolved $\mathrm{CH}_{4}$ concerning contact with the atmosphere. However, dissolved $\mathrm{CH}_{4}$ from decomposition of organic matter can super saturate water to an extent that the ebullitive pathway can periodically overwhelm the diffusive one [7]. Compared to $\mathrm{CO}_{2}$ and $\mathrm{CH}_{4}$ and due to the overlying $78 \% \mathrm{~N}_{2}$ atmosphere, $\mathrm{N}_{2}$ aec emission is potentially more straightforward to model. Solubility of $\mathrm{N}_{2}$ ranges between $18.42 \mathrm{ml} / \mathrm{l}$ (in water at $0{ }^{\circ} \mathrm{C}$ with $0 \%$ salinity) to $6.95 \mathrm{ml} / 1\left(40^{\circ} \mathrm{C}, 40 \%\right.$ ) under one atmosphere total pressure [10]. As one mol of $\mathrm{N}_{2}(28 \mathrm{~g})$ occupies 22.4 liters, the solubility range can be expressed in molarity: $310 \mu \mathrm{mol} / 1$ - $822 \mu \mathrm{mol} / \mathrm{l}$, and the saturation concentration range is $397 \mu \mathrm{mol} / \mathrm{l}$ - $1054 \mu \mathrm{mol} / \mathrm{l}$. For higher altitudes, for instance, at pressure 0.9 atm the solubility range is $279 \mu \mathrm{mol} / 1-740 \mu \mathrm{mol} / \mathrm{l}$ and the saturation concentration range is $357 \mu \mathrm{mol} / 1$ - $949 \mu \mathrm{mol} / \mathrm{l}$. Due to contact with the $78 \% \mathrm{~N}_{2}$ atmosphere, the solubility ranges shown above are actually the background concentrations of dissolved $\mathrm{N}_{2}$ in environmental water bodies. They are close to saturation. Difference between background and saturation are within the range $87 \mu \mathrm{mol} / 1$ - $232 \mu \mathrm{mol} / \mathrm{l}$ under one atmosphere and smaller at higher altitudes, in theory. A higher difference $(250 \mu \mathrm{mol} / \mathrm{l})$ has been measured in prac- 
tice [11] possibly caused by sudden temperature and/or pressure gradients. Therefore, an inflexion in the diffusive emission of $\mathrm{N}_{2}$ aec is expected when dissolved $\mathrm{N}$ concentration in water is as high as $\sim 87$ to $250 \mu \mathrm{mol} / \mathrm{l}$. If a source of $\mathrm{N}_{2}$ (such as denitrification) exists in the water, it will become increasingly unlikely that the $\mathrm{N}_{2}$ thus produced will escape diffusively to the atmosphere and more probable that it will escape ebullitively. A "cat leap" realization led us to infer that, not only does the expected transition in the $\mathrm{N}_{2}$ aec diffusive flux in $\mathrm{N}$-saturated waters exist [12] but that, the $\mathrm{N}_{2}$ aec diffusive emission is modeled fairly well [12] by either of two equations:

$$
y=(640 x) /(180+x)
$$

Alternatively:

$$
y=(700 x) /(320+x)
$$

Actually, Equations (1) and (2) represent diffusively emitted denitrified $\mathrm{N}_{2}$ ( $y$, $\left.\mu \mathrm{mol} \cdot \mathrm{N} \cdot \mathrm{m}^{-2} \cdot \mathrm{h}^{-1}\right)$ as a function of above-background dissolved $\mathrm{N}$ in water $(x$, $\mu \mathrm{mol} \cdot \mathrm{N} \cdot \mathrm{l}^{-1}$ ). Plots of saturation Equations (1) and (2) show how the rate of increase of diffusive denitrified $\mathrm{N}_{2}$ emission with concentration undergoes an inflexion around roughly $\mathrm{x}=87$ to $\mathrm{x}=232 \mu \mathrm{mol} \cdot \mathrm{N} \cdot \mathrm{l}^{-1}$ [12]. These concentrations added to background concentration (310 to 822) are the saturation concentrations (397 and $1054 \mu \mathrm{mol} \cdot \mathrm{N}_{2} \cdot \mathrm{l}^{-1}$ ) of dissolved $\mathrm{N}_{2}$, discussed above. Equations (1) and (2) also show that at limit ${ }_{x \rightarrow \infty}$, diffusive emissions of denitrified $\mathrm{N}_{2}$ can, in theory, increase no more than about $640-700 \mu \mathrm{mol} \mathrm{N} \cdot \mathrm{m}^{-2} \cdot \mathrm{h}^{-1}$. In practice, one order of magnitude higher diffusive emission rates have been measured [12].

Given that diffusive emissions are limited to these maximum values one can expect an increasingly significant ebullitive pathway, especially if there is a source of $\mathrm{N}_{2}$ (e.g. denitrification) in dissolved-N saturated waters. The penalty for disregarding $\mathrm{N}_{2}$ aec (e.g. denitrified $\mathrm{N}_{2}$ ) emitted in bubbles is that the fate of anthropogenic $\mathrm{N}$ will appear to be uncertain [13] [14] because nitrogen will seem to be "missing", in other words, not enough $\mathrm{N}$ output will be found to balance riverine $\mathrm{N}$ inputs and outputs. Several studies observed such imbalances [1] [11] [15]-[22].

The purpose of this work is to model our findings of excess $\mathrm{N}_{2}$ in ebullitive emissions, complement it with the existing diffusive denitrified $\mathrm{N}_{2}$ models described above, and predict denitrified $\mathrm{N}_{2}$ emissions from inland waters with available data on total dissolved $\mathrm{N}$.

\section{Materials and Methods}

\subsection{Studied Sites and Rivers}

Between years 2008 and 2012, we investigated 131 sites distributed among four tropical and two sub-tropical rivers, all in Brazil (Figure 1).

The geographic coordinates, depth, quantity of bubble-samplers (funnels) collectively deployed per site; surface water temperature and atmospheric pressure are in Table A1. Land cover surrounding the surveyed river reaches range 


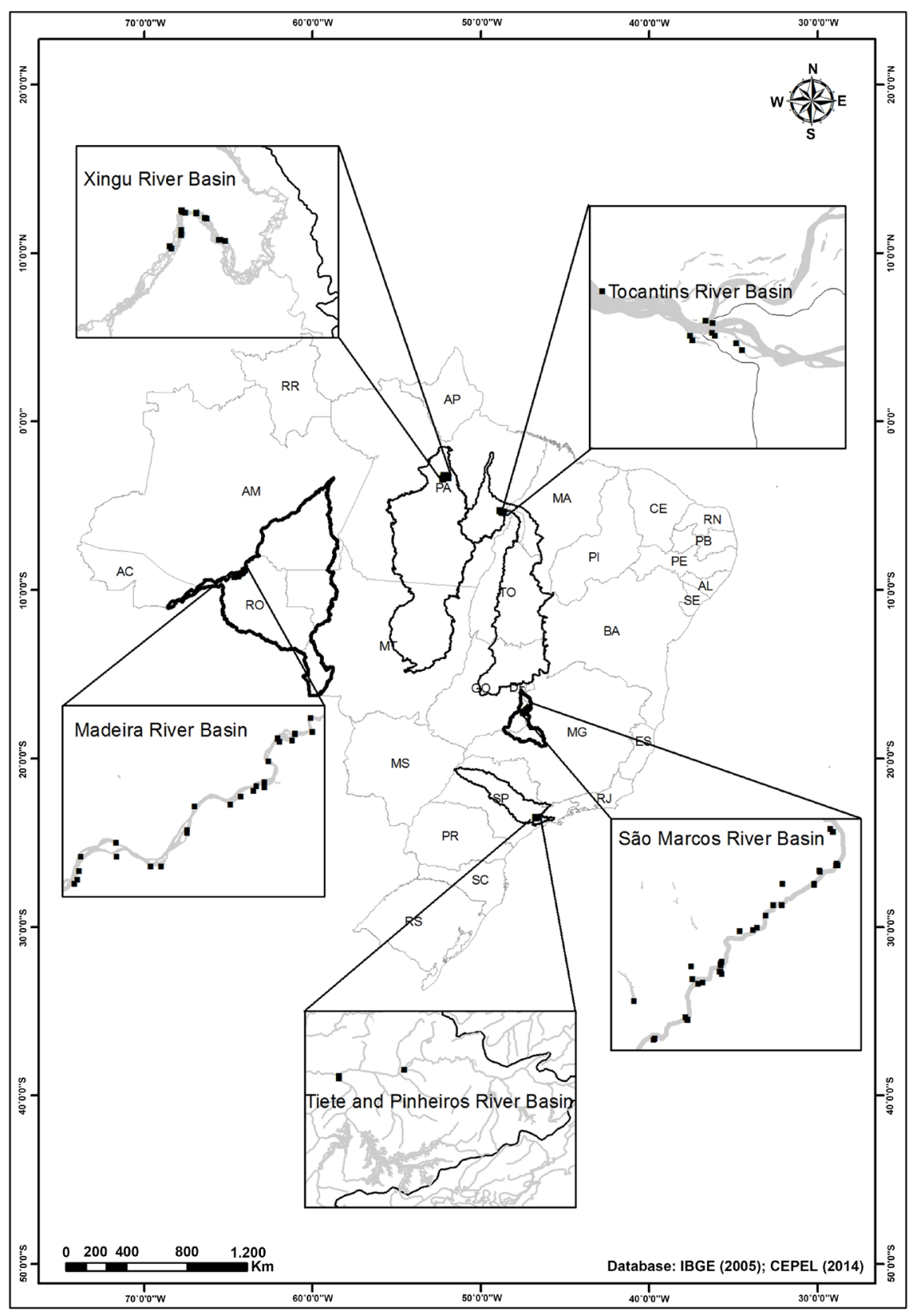

Figure 1. Catchments and sampled sites of the six Brazilian surveyed rivers.

from tropical rainforest typically subjected to periodic floods, to heavily urbanized land.

The purpose was to measure $\mathrm{CH}_{4}$ and $\mathrm{CO}_{2}$ emissions. We studied stretches of the tropical rivers: Xingu (surrounded mainly by tropical rainforest), Tocantins (forest and grassland), Madeira (forest and grassland) and São Marcos (grassland and agricultural land). Also of highly impacted sub-tropical stretches of rivers Tietê and Pinheiros, both surrounded by São Paulo city. Physical and chemical parameters of the investigated reaches are in Table 1.

We surveyed tropical rivers Xingu, Tocantins and Madeira along stretches located in the Brazilian Amazon, near Altamira, Marabá and Porto Velho cities, 
Table 1. Median ( ${ }^{\text {rst }}$ quartile; $3^{\text {rd }}$ quartile) (number of measurements) of physical and chemical parameters of the surveyed areas. Parameter measurements are contemporary with the ebullitive emission measurements reported in this work except for Tocantins River, where we measured bubble emissions in year 2008 but found parameter data only for year 2011.

\begin{tabular}{|c|c|c|c|c|c|c|c|c|c|c|c|c|c|}
\hline \multirow{2}{*}{ River } & \multicolumn{2}{|c|}{$\begin{array}{l}\text { Sampled riverine } \\
\text { stretch/site }\end{array}$} & \multirow{2}{*}{$\begin{array}{c}\text { Dissolved } \\
\text { Oxygen } \\
\left(\mathrm{mg} \cdot 1^{-1}\right)\end{array}$} & \multirow{2}{*}{$\mathrm{pH}$} & \multicolumn{2}{|c|}{$\begin{array}{c}\text { Temperature } \\
\left({ }^{\circ} \mathrm{C}\right)\end{array}$} & \multirow{2}{*}{$\begin{array}{c}\text { Total } \\
\text { Phosphorus } \\
\left(\mathrm{mg} \cdot \mathrm{l}^{-1}\right)\end{array}$} & \multirow[t]{2}{*}{$\begin{array}{c}\text { Ammonium } \\
\left(\mathbf{N H}_{4}^{+}\right)\end{array}$} & \multirow{2}{*}{\multicolumn{2}{|c|}{$\frac{\begin{array}{c}\text { Nitrate } \\
\left(\mathrm{NO}_{3}^{-}\right)\left(\mathrm{NO}_{2}^{-}\right)\end{array}}{\left(\mathrm{mg} \cdot \mathrm{N} \cdot \mathrm{l}^{-1}\right)}$}} & \multirow[t]{2}{*}{$\mathrm{N}_{2}$} & \multirow{2}{*}{$\begin{array}{l}\text { Month and year } \\
\text { of measurement }\end{array}$} & \multirow{2}{*}{ Reference } \\
\hline & $\begin{array}{l}\text { Lat } \\
\left({ }^{\circ} S\right)\end{array}$ & $\begin{array}{l}\text { Long } \\
\left({ }^{\circ} \mathrm{W}\right)\end{array}$ & & & Water & Air & & & & & & & \\
\hline Xingu & $\begin{array}{l}3.5140 \\
\text { to } \\
3.5805\end{array}$ & $\begin{array}{l}51.7109 \\
\quad \text { to } \\
51.7654\end{array}$ & $\begin{array}{c}7.36 \\
{[4.70 ;} \\
7.50] \\
(5)\end{array}$ & $\begin{array}{c}7.00 \\
{[6.94 ;} \\
7.30] \\
(5)\end{array}$ & $\begin{array}{c}30.9 \\
{[30.0 ;} \\
31.6] \\
(5)\end{array}$ & $\cdots$ & $\begin{array}{c}0.068 \\
{[0.021 ;} \\
0.070] \\
(5)\end{array}$ & $\begin{array}{c}<0.0005 \\
{[<0.0005 ;} \\
0.002] \\
(5)\end{array}$ & $\begin{array}{c}0.039 \\
{[0.005} \\
0.156] \\
(5)\end{array}$ & $\ldots^{\mathrm{b}}$ & $\ldots$ & Nov 2008 & [24] \\
\hline Tocantins & 5.1263 & 49.3200 & $\begin{array}{c}9.20 \\
(1)\end{array}$ & $\begin{array}{c}7.70 \\
(1)\end{array}$ & $\begin{array}{c}30.5 \\
(1)\end{array}$ & $\begin{array}{c}29.0 \\
(1)\end{array}$ & $\begin{array}{c}0.032 \\
(1)\end{array}$ & $\begin{array}{l}<\mathrm{DL}^{\mathrm{a}} \\
(1)\end{array}$ & $\begin{array}{l}0.075 \\
(1)\end{array}$ & $\begin{array}{l}<\mathrm{DL} \\
(1)\end{array}$ & $0.488(1)$ & Jun 2011 & \\
\hline Madeira & $\begin{array}{l}8.5056 \\
\text { to } 9.289\end{array}$ & $\begin{array}{l}63.597 \\
\text { to } 64.62\end{array}$ & $\begin{array}{c}6.92 \\
{[5.94 ;} \\
7.69] \\
(42)\end{array}$ & $\begin{array}{c}6.76 \\
{[6.33 ;} \\
7.31] \\
(45)\end{array}$ & $\begin{array}{c}28.1 \\
{[27.8 ;} \\
29.5] \\
(48)\end{array}$ & $\begin{array}{c}27.4 \\
{[26.8 ;} \\
28.6] \\
(50)\end{array}$ & $\begin{array}{c}0.172 \\
{[0.060 ;} \\
0.236] \\
(96)\end{array}$ & $\begin{array}{c}0.020 \\
{[0.017 ;} \\
0.030] \\
(61)\end{array}$ & $\begin{array}{c}0.131 \\
{[0.107 ;} \\
0.171] \\
(61)\end{array}$ & $\begin{array}{c}0.001 \\
{[0.000} \\
0.001] \\
(49)\end{array}$ & $\begin{array}{c}0.60 \\
{[0.47} \\
0.67] \\
(23)\end{array}$ & $\begin{array}{l}\text { May, Aug, Dec } \\
\text { 2011. Feb, Mar } \\
2012\end{array}$ & $\begin{array}{c}\text { BALCAR } \\
\text { databank; } \\
\text { Furnas } \\
\text { Reports }^{\mathrm{d}}\end{array}$ \\
\hline São Marcos & $\begin{array}{l}16.9017 \\
\quad \text { to } \\
17.4430\end{array}$ & $\begin{array}{l}47.1413 \\
\text { to } \\
47.5217\end{array}$ & $\begin{array}{c}7.88 \\
{[7.52} \\
8.65] \\
(32)\end{array}$ & $\begin{array}{c}6.20 \\
{[5.85 ;} \\
6.68] \\
(33)\end{array}$ & $\begin{array}{c}23.4 \\
{[20.9 ;} \\
24.3] \\
(33)\end{array}$ & $\begin{array}{c}27.5 \\
{[25.9 ;} \\
29.0] \\
(32)\end{array}$ & $\begin{array}{c}0.022 \\
{[0.016 ;} \\
0.032] \\
(79)\end{array}$ & $\begin{array}{c}0.017 \\
{[0.009 ;} \\
0.058] \\
(45)\end{array}$ & $\begin{array}{c}0.045 \\
{[0.024} \\
0.071] \\
(45)\end{array}$ & $\begin{array}{c}0.001 \\
{[0.000} \\
0.001] \\
(33)\end{array}$ & $\begin{array}{c}0.050 \\
{[0.023} \\
0.410] \\
(36)\end{array}$ & $\begin{array}{l}\text { Mar, Jun, Oct } \\
2011\end{array}$ & \\
\hline Tietê & $\begin{array}{l}23.5197 \\
\quad \text { to } \\
23.5253\end{array}$ & $\begin{array}{l}46.5592 \\
\quad \text { to } \\
46.7464\end{array}$ & $\begin{array}{c}0.21 \\
{[0.21} \\
0.26] \\
(3)\end{array}$ & $\begin{array}{c}7.2 \\
{[7.2 ;} \\
7.2] \\
(3)\end{array}$ & $\begin{array}{c}20.5 \\
{[20.5 ;} \\
20.8] \\
(3)\end{array}$ & $\begin{array}{c}24.5 \\
{[22.4 ;} \\
25.8] \\
(3)\end{array}$ & $\begin{array}{c}1.71 \\
{[1.58 ; 1.76]} \\
(3)\end{array}$ & $\begin{array}{c}16.1 \\
{[15.8} \\
17.5] \\
(3)\end{array}$ & $\begin{array}{l}<0.2 \\
{[<0.2 ;} \\
<0.2] \\
(3)\end{array}$ & $\begin{array}{c}<0.1 \\
{[<0.1 ;} \\
<0.1] \\
(3)\end{array}$ & $\ldots$ & May 2012 & [23] \\
\hline Pinheiros & 23.5311 & 46.7483 & $\begin{array}{c}<0.21 \\
(1)\end{array}$ & $\begin{array}{l}7.1 \\
(1)\end{array}$ & $\begin{array}{c}21.2 \\
(1)\end{array}$ & $\begin{array}{c}22.1 \\
(1)\end{array}$ & $\begin{array}{c}1.82 \\
(1)\end{array}$ & $\begin{array}{c}17.6 \\
(1)\end{array}$ & $\begin{array}{c}<0.2 \\
(1)\end{array}$ & $\begin{array}{c}<0.01 \\
(1)\end{array}$ & $\cdots$ & May 2012 & \\
\hline
\end{tabular}

${ }^{a}$ Smaller than (unspecified in original report) detection limit. ${ }^{b}$ No existing data. ${ }^{c}$ Month and year this table's parameters were measured. ${ }^{\mathrm{d} B A L C A R}$ Carbon Balance Project private databank and Furnas Reports. Published with permission from Furnas.

respectively. Due to agriculture, only $30 \%$ of tropical river São Marcos catchment's original cerrado (a savanna-type biome) remains. Tietê and Pinheiros rivers are both located in the upper Tietê River basin; their studied sites are on a sub-tropical reach within São Paulo, a city with 11 million inhabitants. São Paulo city's municipal disposal service collects $97 \%$ of total sewage generated and $75 \%$ is treated, but this treatment does not remove $\mathrm{N}$ compounds such as organic $\mathrm{N}$, ammonium, nitrite and nitrate from the effluents discharged into the rivers [23]. Dredged sediment volume from the studied areas in May 2012 for desilting, was $84383 \mathrm{~m}^{3}$ from the $24.5 \mathrm{~km}$ extension $\left(3444 \mathrm{~m}^{3} \cdot \mathrm{km}^{-1}\right)$ of Tietê River canal. And $50387 \mathrm{~m}^{3}$ from the $10.1 \mathrm{~km}$ extension $\left(4988 \mathrm{~m}^{3} \cdot \mathrm{km}^{-1}\right)$ of Pinheiros River canal (2016 email from Waters and Electrical Energy Department of São Paulo City to us).

References [6] and [25] discuss the design of the measurement campaigns.

\subsection{Bubble Sampling}

Bubble emissions were sampled using submerged inverted funnels $(70 \mathrm{~cm} \varnothing \times$ $70 \mathrm{~cm}$ height) using established sampling procedures [5] [6] [25] [26]. Engaged to the narrower opening of the funnel is a bubble-gas collecting vial (typically $500 \mathrm{ml}$ volume). The narrower opening of a deployed funnel is typically about 15 $\mathrm{cm}$ below the water surface. We deployed more than one funnel per site at sites 
closer to river shores and fewer funnels per site at sites closer to river thalwegs, because shallower sites tend to be more ebullitive [25]. Funnels collected ebullitive emissions for about $24 \mathrm{~h}$, unattended, and then were retrieved. At funnel retrieval, transference of the collected ebullitive gas per site into one graduated vial permits total bubble-gas volume measurement. It was not of interest at the time to measure variability of bubble emission among the simultaneously deployed funnels within a same site. After volume measurement, an aliquot was transferred to a $37 \mathrm{ml}$ glass ampoule (made by Construmaq São Carlos) and screw-capped. Bubble-gas sample harvest, total volume measurement and transference into glass ampoules were always done underwater (leaning over the boat), with no exposure of the bubble-gas samples to the atmosphere. Bubble-gas samples $<1.6$ $\mathrm{ml}$ were discarded because, although sufficient for chromatographic analyses, they were insufficient for purging and transference. When sampling was done for the day and the boat returned to shore, the glass ampoules containing the samples were immediately taken to our field portable-laboratory for chromatographic analysis.

\subsection{Bubble-Gas Sample Transference into a Syringe}

In the laboratory, bubble-gas was transferred from the glass ampoule into a syringe (BD Ultra-fine ${ }^{\mathrm{TM}} 12.5 \mathrm{~mm}$ needle-length, purchased over the counter) using a $0.6 \mathrm{ml}$ volume transfer equipment. This equipment consisted of $50 \mathrm{~cm}$ length tubing and a glass bulb, previously purged with sample. Tubing consisted of stainless steel tube $1.5 \mathrm{~mm}$ outside diameter $(\mathrm{OD}) \times 1.0 \mathrm{~mm}$ inner diameter (ID) $\times 30 \mathrm{~cm}$ length, and PVC tubing $2.0 \mathrm{~mm}$ OD $\times 1.3 \mathrm{~mm}$ ID $\times 20 \mathrm{~cm}$ length. Connected to the PVC tubing was a small glass bulb, sealed with a small plug made with stationery-shop-purchased white vinyl eraser. (Sample transferring setup image is available at

http://www.construmaq.ind.br/produtos/bulbo-de-vidro-selado-com-rolha-de-b orracha-e-inserido-em-mangueira-flexivel/)

\subsection{Chromatographic Analyses}

Samples were chromatographically analyzed for $\mathrm{CH}_{4}$ and $\mathrm{CO}_{2}$, within the first 24 hours after being harvested. Oxygen and $\mathrm{N}_{2}$ peaks elute from the Molecular Sieve chromatographic column prior to methane's (peak area is proportional to gas concentration). The $\mathrm{O}_{2}$ peak is in fact an $\mathrm{O}_{2}+\mathrm{Ar}$ peak because these two gases elute together; chromatograms showed them as one combined peak. We used a Molecular Sieve 5A filled stainless steel chromatographic column of $3.2 \mathrm{~mm}$ $(\mathrm{OD}) \times 1.6 \mathrm{~mm}(\mathrm{ID}) \times 1.95 \mathrm{~m}$ length and a thermal conductivity detector $(\mathrm{TCD})$ chromatograph manufactured by Construmaq São Carlos. Carrier gas was hydrogen $\left(\mathrm{H}_{2}\right)$. Injector, column and detector operated at room temperature. Samples were injected by hand using the BD syringe mentioned above. Injected gas volumes were 0.1 milliliter $(100 \mu \mathrm{l})$. Variability (average ratio of standard deviation divided by average peak area of 3 peaks) was $\pm 1.5 \% \mathrm{O}_{2}$ and $\pm 1.2 \% \mathrm{~N}_{2}$. Detection limits were $0.5 \% \mathrm{O}_{2}$ and $1 \% \mathrm{~N}_{2}$. Oxygen and Ar elute together from the 
Molecular Sieve 5A column as one combined peak, followed by the $\mathrm{N}_{2}$ peak and $\mathrm{CH}_{4}$ peak. Reference [27] briefly mentions that $5 \%$ of the $\mathrm{O}_{2}+\mathrm{Ar}$ peak is Ar.

\section{5. $\mathrm{N}_{2}$ aec Bubble-Emission Calculation Method}

Consider data from the third line of data in Table A1(a). After a period of 22.33 sampling hours, four funnels at $5.5 \mathrm{~m}$ depth Xingu River site $3.3118^{\circ} \mathrm{S}$ $52.1960^{\circ} \mathrm{W}$, collectively collected $520 \mathrm{ml}$ of gas. Chromatographic analysis of an aliquot of the collected volume resulted $4.89 \% \mathrm{O}_{2}+\mathrm{Ar}$ and $26.5 \% \mathrm{~N}_{2}$. The bubble sample had $0.2445 \% \operatorname{Ar}\left(5 \%\right.$ of the $\mathrm{O}_{2}+\mathrm{Ar}$ mixture). Environmental $\mathrm{N}_{2}$, in the bubble sample was $0.2445 \% \mathrm{Ar} \times 78.1 \% \mathrm{~N}_{2} / 0.93 \% \mathrm{Ar}=20.5 \%$. Emitted $\mathrm{N}_{2}$ aec concentration, in bubble sample was $26.5 \%-20.5 \%=6.0 \%$. Emitted $\mathrm{N}_{2}$ aec volume, in the bubble sample was:

$$
\frac{6.0 \% \mathrm{~N}_{2} \mathrm{aec} \times 520 \mathrm{ml}}{100 \% \times 1000 \mathrm{ml} \cdot 1^{-1}}=0.03121
$$

Emitted $\mathrm{N}_{2}$ aec-N mass, in bubble sample was:

$$
\frac{747.0 \mathrm{mmHg} \times 0.0312 \mathrm{l} \times 28 \mathrm{~g} \cdot \mathrm{N}_{2} \mathrm{aec}-\mathrm{N} \cdot \mathrm{mol}^{-1} \times 1000 \mathrm{mg} \cdot \mathrm{g}^{-1}}{62.41 \cdot \mathrm{mmHg} \cdot \mathrm{mol}^{-1} \cdot \mathrm{K}^{-1} \times 301.7 \mathrm{~K}}=34.6 \mathrm{mg}
$$

Funnel area was $0.3848 \mathrm{~m}^{2}$. Rate of emitted $\mathrm{N}_{2}$ aec-N from site $3.3118^{\circ} \mathrm{S}$ $52.1960^{\circ} \mathrm{W}$ was:

$$
\frac{34.6 \mathrm{mg} \times 24 \mathrm{~h} \cdot \mathrm{d}^{-1}}{4 \times 0.3848 \mathrm{~m}^{2} \times 22.33 \mathrm{~h}}=24.1 \mathrm{mg} \cdot \mathrm{m}^{-2} \cdot \mathrm{d}^{-1}
$$

Variability is $1.9 \%\left(\sqrt{1.5^{2}+1.2^{2}}\right)$. Had the collected volume been $1.6 \mathrm{ml}$ rather than $520 \mathrm{ml}$, then, the minimum detectable ebullitive $\mathrm{N}_{2}$ aec emission in this case would have been $74.2 \mu \mathrm{g} \mathrm{N} \mathrm{N}_{2}$ aec- $\mathrm{N} \cdot \mathrm{m}^{-2} \cdot \mathrm{d}^{-1}$.

\section{Results}

All six sampled rivers were sources of ebullitive $\mathrm{N}_{2} \mathrm{aec}$ (Table A1, Appendix). Two out of the 131 collected samples lacked data (e.g. bubble emission volume data) to calculate emission; even so, those 2 samples were chromatographically analyzed (Table A1(b) and Table A1(d)). From the 129 ebullitive emission samples which provided $\mathrm{N}_{2}$ emission rates, 19 had insufficient volume $(<1.6 \mathrm{ml})$ for transfer-tube purging and chromatographic analyses and were labeled "zero emission" (Table A1(a) and Table A1(b)). Thirty-eight samples had sub-environmental $\mathrm{N}_{2}$ concentrations (Tables A1(a)-(d)). These 38 samples resulted in negative emission rates of $\mathrm{N}_{2}$ aec. They were also considered "zero emission", for the purpose of $\mathrm{N}_{2}$ aec emission quantification. Therefore, less than half (44\%) of the 129 samples yielded zero $\mathrm{N}_{2}$ aec ebullitive emission either because of too small sample volume or due to a negative emission result. Table 2 summarizes bubble emission measurement results.

\subsection{Ebullitive $\mathrm{N}_{2}$ aec Emission Model}

Ebullitive $\mathrm{N}_{2}$ eac emission increased with dissolved (reactive + inert) $\mathrm{N}$ concentration in water (Figure 2). Using median emission rate per river (Figure 2), the 
Table 2. Ebullitive $\mathrm{N}_{2}$ aec-N medians given in both emission rate units used in this work, and dissolved $\mathrm{N}$ concentration in the studied rivers.

\begin{tabular}{ccccc}
\hline River & $\begin{array}{c}\text { Quantity of } \\
\text { measurements }\end{array}$ & \multicolumn{2}{c}{$\begin{array}{c}\text { Median } \mathrm{N}_{2} \text { aec-N ebullitive emission } \\
\text { (first quartile; third quartile) }\end{array}$} & $\begin{array}{c}\text { Median dissolved N } \\
\text { concentration } \\
\left(\mu \mathrm{mol} \cdot \mathrm{N} \cdot \mathrm{l}^{-1}\right)\end{array}$ \\
\cline { 3 - 4 } Xingu & 38 & $0(0 ; 1.50)$ & $0(0 ; 4.44)$ & 2.79 \\
São Marcos & 37 & $0.54(0 ; 9.73)$ & $1.6(0 ; 28.8)$ & 8.07 \\
Tocantins ${ }^{\mathrm{a}}$ & 23 & $2.92(0 ; 10.8)$ & $8.7(0 ; 32.2)$ & 40.2 \\
Madeira & 26 & $40.5(15.8 ; 79.6)$ & $121(47.2 ; 238)$ & 53.7 \\
Tietê & 3 & $355(293 ; 405)$ & $1057(872 ; 1206)$ & 1150 \\
Pinheiros & 2 & $934(858 ; 1011)$ & $2780(2554 ; 3009)$ & 1257 \\
\hline
\end{tabular}

${ }^{a}$ Tocantins River data are not used to model dinitrogen emission because ebullitive emission (measured in 2008) and dissolved $\mathrm{N}$ concentration (measured in 2011) are not contemporary.

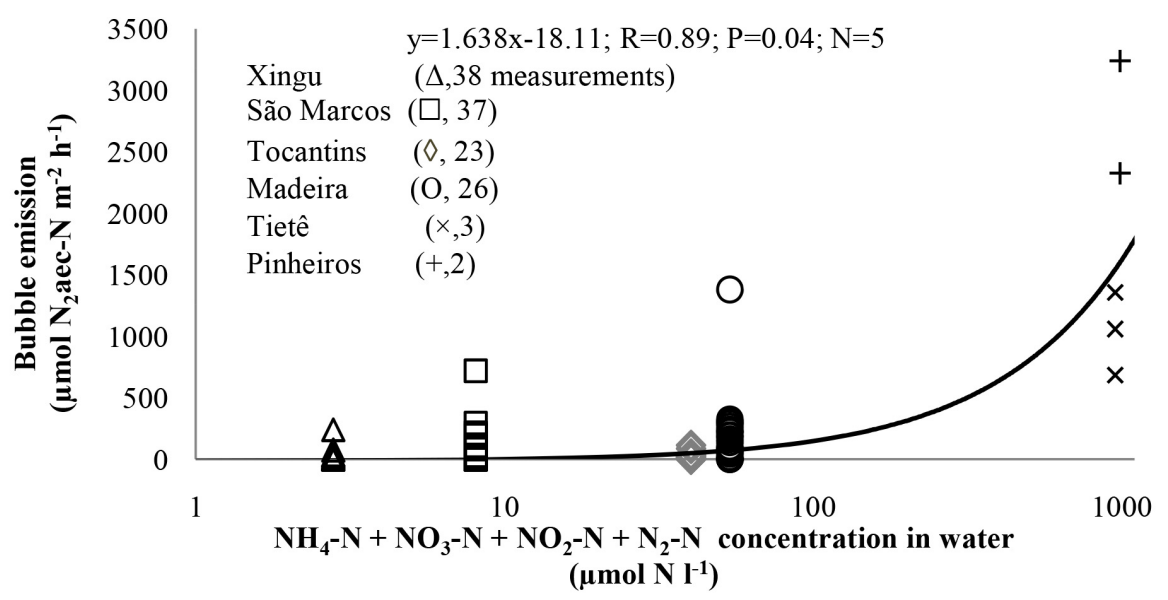

Figure 2. Data markers distant from clusters represent measured outliers. Table A1's measured ebullitive $\mathrm{N}_{2}$ aec emission rates (y) in unit $\mu \mathrm{mol} \cdot \mathrm{N}_{2} \mathrm{aec}-\mathrm{N} \cdot \mathrm{m}^{-2} \cdot \mathrm{h}^{-1} \quad(1$ $\mathrm{mg} \cdot \mathrm{N}_{2}$ aec $-\mathrm{N} \cdot \mathrm{m}^{-2} \cdot \mathrm{d}^{-1} \cong 3 \mu \mathrm{mol} \cdot \mathrm{N}_{2}$ aec $\left.-\mathrm{N} \cdot \mathrm{m}^{-2} \cdot \mathrm{h}^{-1}\right)$, plotted against dissolved $\mathrm{N}\left(\mathrm{NH}_{4}-\mathrm{N}+\right.$ $\mathrm{NO}_{3}-\mathrm{N}+\mathrm{NO}_{2}-\mathrm{N}+\mathrm{N}_{2}-\mathrm{N}, \mathrm{x}$ ) median concentration (Table 1). Linear fit is curved "up" due to the $\log -\mathrm{X}$ plot, and was calculated with median emission from Xingu (0 $\mu \mathrm{mol} \cdot \mathrm{N}_{2}$ aec- $\mathrm{N} \cdot \mathrm{m}^{-2} \cdot \mathrm{h}^{-1}$ ), São Marcos (1.6), Madeira (121), Tietê (1057) and Pinheiros (2780), as functions of dissolved $\mathrm{N}$ concentration $(2.79 ; 8.07 ; 53.7 ; 1150 ; 1257 \mu \mathrm{M}$ N, respectively). Tocantins' 23 rates $(\diamond)$ median $\left(8.7 \mu \mathrm{mol} \cdot \mathrm{N}_{2} \mathrm{aec}-\mathrm{N} \cdot \mathrm{m}^{-2} \cdot \mathrm{h}^{-1}\right)$ was not used to calculate linear fit because it is unlikely that year 2011's dissolved $\mathrm{N}, 40.2 \mu \mathrm{M} \mathrm{N}$, was valid also back in year 2008, when $\mathrm{N}_{2}$ aec bubble emission from Tocantins River was measured.

dependence of bubble emission on concentration is best (high R/small P) described by the first-order equation:

$$
y=1.638 x-18.11
$$

where $y$ is $\mathrm{N}_{2}$ aec bubble emission $\left(\mu \mathrm{mol} \cdot \mathrm{N}_{2}\right.$ aec $\left.-\mathrm{N} \cdot \mathrm{m}^{-2} \cdot \mathrm{h}^{-1}\right)$ and $\mathrm{x}$ is total dissolved (reactive + inert) $\mathrm{N}$ concentration in water $\left(\mu \mathrm{mol} \cdot \mathrm{N} \cdot \mathrm{l}^{-1}\right)$. As bubble growth is a function of dissolved gas concentration in water [28], it is pertinent to know (besides reactive $\mathrm{N}$ concentration) how much dissolved $\mathrm{N}_{2}$ is in the studied water. 
If investigated $\mathrm{N}_{2}$ emissions from swine farms [29] hold for rivers too then, for dissolved $\mathrm{N}$ concentrations $>1200 \mu \mathrm{mol} \cdot \mathrm{N} \cdot \mathrm{l}^{-1}$ ebullitive emissions increase at a smaller rate and, rather than by Equation (6), are better described by:

$$
y=0.4300 x+1430.7
$$

\subsection{Total $\mathrm{N}_{2}$ aec Emission Model}

For a given total dissolved $\mathrm{N}$ concentration in water, the ebullitive emission (Equation (6) if dissolved N concentrations $<1200 \mu \mathrm{mol} \cdot \mathrm{N} \cdot \mathrm{l}^{-1}$ and Equation (7) if concentrations $>1200 \mu \mathrm{mol} \cdot \mathrm{N} \cdot \mathrm{l}^{-1}$ ) plus the diffusive emission (Equation (1) or (2)) results total $\mathrm{N}_{2}$ aec emission (Figure 3).

Actually, diffusive emissions were originally [12] plotted against dissolved $\mathrm{NO}_{3}-\mathrm{N}$, which, for lack of more data on dissolved $\mathrm{N}$, we assume roughly represents total dissolved N. The ready-to-use version of our model (Table 3) will be used while working through the five case studies.

\subsection{Partition between Ebullitive and Diffusive $\mathrm{N}_{2}$ aec Emissions}

The ebullitive:diffusive partition ratio changes radically with increasing dissolved $\mathrm{N}$. In river water with small concentrations $\left(<11 \mu \mathrm{mol} \cdot \mathrm{N} \cdot \mathrm{l}^{-1}\right)$ of dissolved $\mathrm{N}, \mathrm{N}_{2}$ aec ebullitive emissions are not significant and diffusive emissions predominate (Figure 3(a), Table 3 and Figure 4).

In waters with $>11 \mu \mathrm{mol} \cdot \mathrm{N} \cdot \mathrm{l}^{-1}$ ebullitive $\mathrm{N}_{2}$ aec emissions increase steadily, along with the diffusive ones. At concentrations between $140-240 \mu \mathrm{mol} \cdot \mathrm{N} \cdot \mathrm{l}^{-1}$ ebullitive rate equal diffusive rate emissions (Figure 3(b) and Figure 4). At concentrations $>250 \mu \mathrm{mol} \cdot \mathrm{N} \cdot \mathrm{l}^{-1}$, ebullitive will tend to be higher than diffusive emissions (Figure 3(b) and Figure 3(c), and Figure 4). In the concentration range $300-1200 \mu \mathrm{mol} \cdot \mathrm{N} \cdot \mathrm{l}^{-1}$ water is supersaturated with dissolved $\mathrm{N}$ and bubble emissions continue to increase, while diffusive emissions eventually saturate at $640-700 \mu \mathrm{mol} \cdot \mathrm{N}_{2} \mathrm{aec}-\mathrm{N} \cdot \mathrm{m}^{-2} \cdot \mathrm{h}^{-1}$ (Figure $3(\mathrm{c}$ ) and Figure 4 ). In waters with dissolved $\mathrm{N}$ concentration $>1200 \mu \mathrm{mol} \cdot \mathrm{N} \cdot \mathrm{l}^{-1}$ ebullitive emissions predominate (Figure 3(d) and Figure 4).

In addition, ebullitive $\mathrm{N}_{2}$ aec emission correlates significantly with $\mathrm{CH}_{4}$ ebullitive emission ( $\mathrm{R}=0.96 ; \mathrm{P}=0.002 ; \mathrm{n}=11$; data from Table $\mathrm{A} 1$; graph not shown) and $\mathrm{CO}_{2}$ ebullitive emission $(\mathrm{R}=0.94 ; \mathrm{P}=0.005 ; \mathrm{n}=11$; data from Table A1; graph not shown), suggesting that the production of $\mathrm{N}_{2} \mathrm{aec}$ is associated with decomposition of organic matter.

\section{Case Study Application}

The following five case studies use the findings here reported to estimate $\mathrm{N}_{2}$ aec emission across aquatic environments.

\subsection{Case Study 1: The "Missing" Nitrogen}

Approximately $50 \%$ of the net anthropogenic $\mathrm{N}$ input was unaccounted for in a watershed $\mathrm{N}$ budget, which included diffusive but not ebullitive emission [22]. 


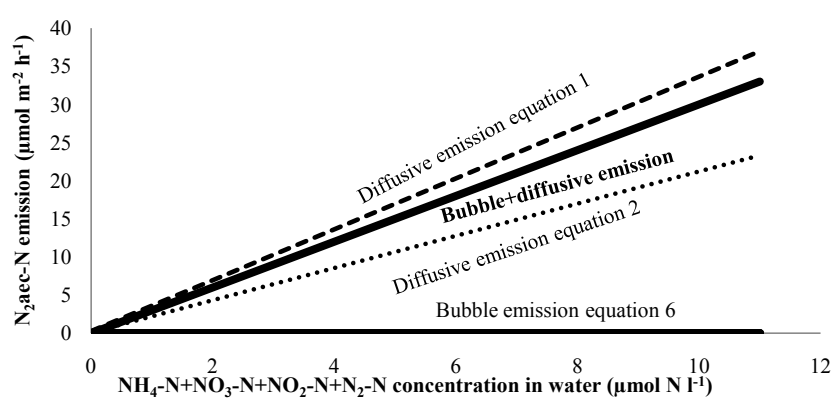

(a)

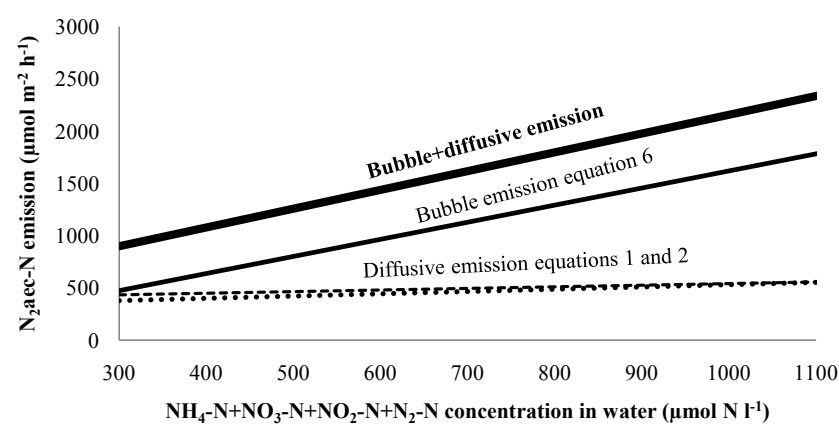

(c)

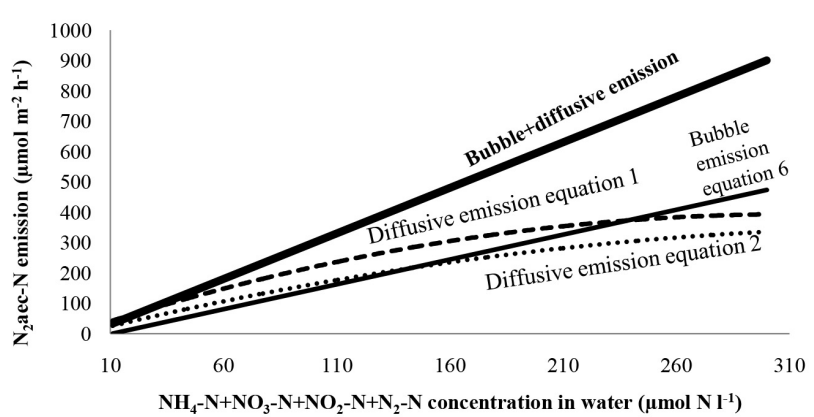

(b)

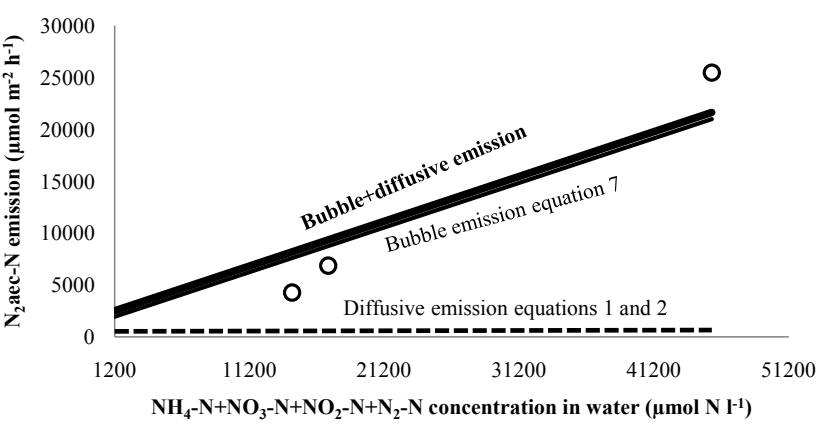

(d)

Figure 3. (a), (b), (c) and (d) Graphical representations of ebullitive (bubble), diffusive, and total (bubble + diffusive) $\mathrm{N}_{2}$ aec water-air emission as a function of total $\mathrm{N}$ concentration in water. Circles in Figure 3(d) are reference [29]'s denitrified $\mathrm{N}_{2}$ bubble emission data from swine farms.

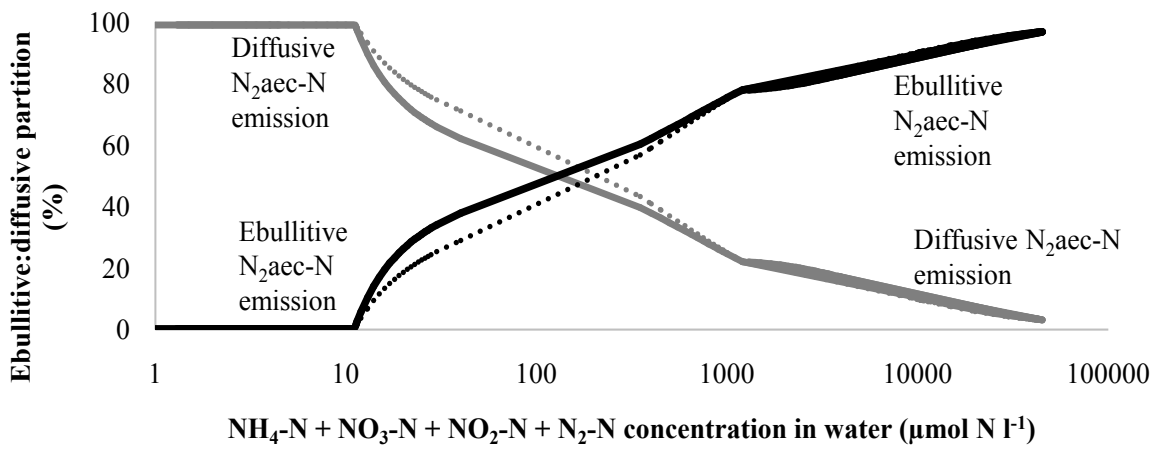

Figure 4. The "scissors-like" ebullitive:diffusive partition. $\mathrm{N}_{2}$ aec emission percentage (\%, $\mathrm{y})$ as a function of dissolved total $\mathrm{N}$ in water $\left(\mu \mathrm{mol} \cdot \mathrm{N} \cdot \mathrm{l}^{-1}, \mathrm{x}\right)$.

Figure 4 shows that ratio $63 \%$ - 65\% ebullitive/37\% - 35\% diffusive $\mathrm{N}$ emission is predicted for stream waters with $500 \mu \mathrm{mol} \cdot \mathrm{N} \cdot \mathrm{l}^{-1}$ such as those studied. Albeit a higher ebullitive loss (63\% to $65 \%)$ than what was actually missing ( $50 \%)$, this still shows that the fate of the missing $\mathrm{N}$ can be explained by ebullitive losses. See "Conclusion".

\subsection{Case Study 2: Nitrogen-Removal Rates}

Nitrogen-removal rates were measured [30] with the membrane inlet mass spectrometry (MIMS) technique. Using the nitrate concentration data from Sugar Creek and Iroquois River [30] and our model, we find: 
Table 3. $\mathrm{N}_{2}$ aec emission ( $\mu \mathrm{mol} \cdot \mathrm{N}_{2}$ aec-N$\cdot \mathrm{m}^{-2} \cdot \mathrm{h}^{-1}$, y) as a function of dissolved $\mathrm{N}$ concentration $\left(\mu \mathrm{mol} \cdot \mathrm{N} \cdot \mathrm{l}^{-1}\right.$, $\left.\mathrm{x}\right)$ in water, within the 0 to $45,500 \mu \mathrm{mol} \cdot \mathrm{N} \cdot \mathrm{l}^{-1}$ concentration range.

\begin{tabular}{|c|c|c|c|}
\hline $\begin{array}{l}\text { Total dissolved } \mathrm{N} \text { concentration } \\
\text { range }\left(\mu \mathrm{mol} \cdot \mathrm{N} \cdot \mathrm{l}^{-1}, \mathrm{x}\right)\end{array}$ & $\begin{array}{c}\text { Ebullitive emission } \\
\left(\mu \mathrm{mol} \cdot \mathrm{N}_{2} \text { aec }-\mathrm{N} \cdot \mathrm{m}^{-2} \cdot \mathrm{h}^{-1}, \mathrm{y}\right)(\mathrm{A})\end{array}$ & $\begin{array}{c}\text { Diffusive emission } \\
\left(\mu \mathrm{mol} \cdot \mathrm{N}_{2} \text { aec }-\mathrm{N} \cdot \mathrm{m}^{-2} \cdot \mathrm{h}^{-1}, \mathrm{y}\right)(\mathrm{B})\end{array}$ & $\begin{array}{c}\text { Total emission } \\
\left(\mu \mathrm{mol} \cdot \mathrm{N}_{2} \text { aec- } \mathrm{N} \cdot \mathrm{m}^{-2} \cdot \mathrm{h}^{-1}, \mathrm{y}\right)(\mathrm{A}+\mathrm{B})\end{array}$ \\
\hline $0-11$ & 0 & \multirow{4}{*}{$\begin{array}{c}\text { Either } \\
y=(640 x) /(180+x) \\
\text { or } \\
y=(700 x) /(320+x)\end{array}$} & $y \approx 3 x$ \\
\hline $11.1-300$ & $y=1.638 x-18.11$ & & $y \approx 3 x$ \\
\hline $300-1200$ & $y=1.638 x-18.11$ & & $y=1.797 x+360.6$ \\
\hline $1200-45,500$ & $y=0.4300 x+1430.7$ & & $y=0.4315 x+1999.2$ \\
\hline
\end{tabular}

1) How denitrified $\mathrm{N}_{2}$-emission is partitioned between ebullition and diffusion. For example, we estimated 83 to $123 \mu \mathrm{mol} \cdot \mathrm{N} \cdot \mathrm{m}^{-2} \cdot \mathrm{h}^{-1}$ diffusive emission (Table 4, line "Sugar Creek Sep 1999", column "Diffusive estimated by us"); and $52 \mu \mathrm{mol} \cdot \mathrm{N} \cdot \mathrm{m}^{-2} \cdot \mathrm{h}^{-1}$ ebullitive emission (column "Ebullitive estimated by us");

2) Significant discrepancy between predicted and measured rates for the high dissolved $[\mathrm{N}]$ range. For example, in June 2001 Sugar Creek's sampled waters had the relatively high dissolved $\mathrm{N}$ concentration $1096 \mu \mathrm{mol} / \mathrm{l}$ (Table 4). While a relatively small denitrification rate range $290 \pm 151 \mu \mathrm{mol} \cdot \mathrm{N} \cdot \mathrm{m}^{-2} \cdot \mathrm{h}^{-1}$ (Table 4) was found, we estimated eight times higher rates 2319 to $2327 \mu \mathrm{mol} \cdot \mathrm{N} \cdot \mathrm{m}^{-2} \cdot \mathrm{h}^{-1}$ (Table 4).

\subsection{Case Study 3: Nitrogen Budget}

Estimation of total annual $\mathrm{N}_{2}$ emitted and $\mathrm{N}$ buried in a subtropical river reservoir (Xipi) in southeast China with $8.5 \mathrm{~km}$ channel length and mean width of $125 \mathrm{~m}$. For Xipi an annual $80 \cdot 10^{3} \mathrm{~kg}$ gaseous $\mathrm{N}$ total emission was estimated [31], while we obtained $88 \cdot 10^{3} \mathrm{~kg} \cdot \mathrm{N}$ ("TOTAL" line, Table 5). However, assuming permanent carbon sedimentation median rate $78 \mathrm{mg} \cdot \mathrm{C} \cdot \mathrm{m}^{-2} \cdot \mathrm{d}^{-1}[32]$ and that the Redfield ratio 6.625 (106 C:16 N) roughly holds, we estimate sedimentation rate is:

$$
78 / 6.625=11.77 \mathrm{mg} \cdot \mathrm{N} \cdot \mathrm{m}^{-2} \cdot \mathrm{d}^{-1}
$$

In addition, annual buried $\mathrm{N}$ is:

$$
\begin{aligned}
& 8500 \mathrm{~m} \times 125 \mathrm{~m} \times\left(11.77 \mathrm{mg} \cdot \mathrm{N} \cdot \mathrm{m}^{-2} \cdot \mathrm{d}^{-1}\right) \times(1 \mathrm{~g} / 1000 \mathrm{mg}) \\
& \times(1 \mathrm{~kg} / 1000 \mathrm{~g}) \times(365 \mathrm{~d} / \mathrm{yr})=456410^{3} \mathrm{~kg} \cdot \mathrm{N} \cdot \mathrm{yr}^{-1}
\end{aligned}
$$

It follows that the total annual retention $(88.260+4.564) \times 10^{3} \approx 93 \times 10^{3} \mathrm{~kg}$ estimated by us, is equal to $93 \times 10^{3} \mathrm{~kg} \cdot \mathrm{N}$ annual retention of dissolved inorganic $\mathrm{N}$, estimated by using a different approach [31].

\subsection{Case Study 4: $\mathrm{N}_{2}$ Emission Estimation}

The Jiulong River is a large agricultural river in southeast China. We use $\mathrm{N}$ concentration data (Table 6) to estimate its $\mathrm{N}_{2}$ water-air emissions. These estimates are then compared to those of co-workers [33]:

1) The North River median area-weighted $\mathrm{N}_{2}$ flux range $812-873 \mu \mathrm{mol} \cdot \mathrm{N} \cdot \mathrm{m}^{-2} \cdot \mathrm{h}^{-1}$ (estimated by us, Table 6) expressed in $\mathrm{kg} \cdot \mathrm{N} \cdot \mathrm{ha}^{-1} \cdot \mathrm{yr}^{-1}$ is: 
Table 4. Source data (from reference [30]) are shown in bold font.

\begin{tabular}{|c|c|c|c|c|c|c|c|}
\hline \multirow[b]{2}{*}{ Date } & \multirow{2}{*}{$\begin{array}{c}\mathrm{NO}_{3} \\
(\mu \mathrm{mol} \mathrm{N} / \mathrm{l}) \\
(\mathrm{A})\end{array}$} & \multicolumn{4}{|c|}{ Denitrified $\mathrm{N}_{2}\left(\mu \mathrm{mol} \cdot \mathrm{N} \cdot \mathrm{m}^{-2} \cdot \mathrm{h}^{-1}\right)$} & \multicolumn{2}{|c|}{$\mathrm{N}\left(\% \mathrm{~d}^{-1}\right)^{\mathrm{c}}$} \\
\hline & & $\begin{array}{c}\text { Diffusive } \\
\text { estimated by us } \\
\text { (B) }\end{array}$ & $\begin{array}{c}\text { Ebullitive } \\
\text { estimated by us } \\
\text { (C) }\end{array}$ & $\mathrm{B}+\mathrm{C}$ & Smith et al. [30] & Estimated by us & $\begin{array}{c}\text { Measured by } \\
\text { Smith et al. } \\
{[30]}\end{array}$ \\
\hline \multicolumn{8}{|c|}{ Sugar Creek } \\
\hline Sep 1999 & 43 & $83-123$ & 52 & $135-176$ & $76 \pm 78$ & $25-32$ & 14 \\
\hline May 2000 & 717 & $484-512$ & 1156 & $1640-1668$ & $1277 \pm 1201$ & $14-14$ & 11 \\
\hline June 2001 & 1096 & $542-550$ & 1777 & $2319-2327$ & $290 \pm 151$ & $13-13$ & 2 \\
\hline Sep 2001 & 135 & $208-274$ & 203 & $411-477$ & $327 \pm 300$ & $24-28$ & 19 \\
\hline \multicolumn{8}{|c|}{ Iroquois River } \\
\hline Sep 1999 & 42 & $81-121$ & 51 & $132-172$ & $100 \pm 67$ & $9-12$ & 7 \\
\hline May 2000 & 840 & $507-527$ & 1358 & $1865-1885$ & $711 \pm 305$ & $5-5$ & 2 \\
\hline Sep 2001 & 79 & $139-195$ & 111 & $250-307$ & $181 \pm 261$ & $23-28$ & 17 \\
\hline
\end{tabular}

${ }^{a}$ Using Equations (2) and (1). E.g.: $[(700 \times \mathrm{A}) /(320+\mathrm{A})]$ and $[(640 \times \mathrm{A}) /(180+\mathrm{A})] .{ }^{\mathrm{b}}$ Using Equation $(6)$. E.g.: $[(1.638 \times \mathrm{A})-18.11] .{ }^{\mathrm{c}} \mathrm{N}\left(\% \mathrm{~d}^{-1}\right)$ is the percentage of water-column nitrate removed by denitrification. E.g.: $((135$ to 176$) \times 14) / 76=(25$ to 32$) \% \mathrm{~N} \cdot \mathrm{d}^{-1}$.

Table 5. Source data (from reference [31]'s Figure 5) are shown in bold font.

\begin{tabular}{|c|c|c|c|c|c|c|c|}
\hline Time interval & $\begin{array}{c}\text { DIN } \\
\left(\mu \mathrm{mol} \cdot \mathrm{N} \cdot \mathrm{l}^{-1}\right) \\
(\mathrm{A})\end{array}$ & $\begin{array}{c}\Delta \mathrm{N}_{2}-\mathrm{N} \\
\left(\mu \mathrm{mol} \cdot \mathrm{l}^{-1}\right)^{\mathrm{a}} \\
(\mathrm{B})\end{array}$ & $\begin{array}{c}\Delta \mathrm{N}_{2} \mathrm{O}-\mathrm{N} \\
\left(10^{-3} \mu \mathrm{mol} \cdot \mathrm{l}^{-1}\right) \\
(\mathrm{C})\end{array}$ & $\begin{array}{c}\text { Total } \\
\left(\mu \mathrm{mol} \cdot \mathrm{N} \cdot \mathrm{l}^{-1}\right) \\
\mathrm{A}+\mathrm{B}+\mathrm{C}\end{array}$ & $\begin{array}{c}\text { Our estimate of } \\
\text { emitted } \mathrm{N}_{2} \\
\left(\mu \mathrm{mol} \cdot \mathrm{N} \cdot \mathrm{m}^{-2} \cdot \mathrm{h}^{-1}\right)\end{array}$ & $\begin{array}{l}\text { Number of } \\
\text { interpolated days }\end{array}$ & $\begin{array}{c}\text { Our estimate for } \\
\text { total } \mathrm{N}_{2} \text { emitted } \\
(\mathrm{kg} \cdot \mathrm{N})\end{array}$ \\
\hline May Jun 2012 & 120 & 12 & 5.727 & 132.0057 & $396.0172^{\mathrm{b}}$ & 61 & $8624.066^{\mathrm{d}}$ \\
\hline Jul Aug Sep 2012 & 150 & 70 & 12.727 & 220.0127 & $660.0382^{\mathrm{b}}$ & 92 & $21,678.29$ \\
\hline $\begin{array}{c}\text { Oct Nov Dec } 2012 \\
\text { Jan } 2013\end{array}$ & 200 & 16 & 13.364 & 216.0134 & $648.0401^{\mathrm{b}}$ & 123 & $28,456.09$ \\
\hline Feb Mar Apr 2013 & 300 & 16 & 31.818 & 316.0318 & $928.5091^{c}$ & 89 & $29,501.52$ \\
\hline & & & TOTAL & & & & $88,259.97$ \\
\hline
\end{tabular}

${ }^{\mathrm{a}}$ Dissolved $\mathrm{N}_{2}$, in excess of environmental (background) dissolved $\mathrm{N}_{2} \cdot{ }^{\mathrm{b}} 3 \times(\mathrm{A}+\mathrm{B}+\mathrm{C})$, see Table 3. ${ }^{\mathrm{c}}[(1.797 \times(\mathrm{A}+\mathrm{B}+\mathrm{C}))+360.6]$, see Table 3. ${ }^{\mathrm{d}} 8500 \mathrm{~m}$ $\times 125 \mathrm{~m} \times\left(396.0172 \mu \mathrm{mol} \cdot \mathrm{N} \cdot \mathrm{m}^{-2} \cdot \mathrm{h}^{-1}\right) \times\left(24 \mathrm{~h} \cdot \mathrm{d}^{-1}\right) \times(61 \mathrm{~d}) \times(1 \mathrm{mmol} / 1000 \mu \mathrm{mol}) \times(1 \mathrm{~mol} / 1000 \mathrm{mmol}) \times(14 \mathrm{~g} / \mathrm{mol}) \times(1 \mathrm{~kg} / 1000 \mathrm{~g})=8624.066 \mathrm{~kg} \mathrm{~N} \mathrm{per} 61$ days.

$$
\begin{aligned}
& \left(812 \mu \mathrm{mol} \cdot \mathrm{N} \cdot \mathrm{m}^{-2} \cdot \mathrm{h}^{-1}\right) \times\left(10^{-6} \mathrm{~mol} \cdot \mu \mathrm{mol}^{-1}\right) \times\left(100 \mathrm{~m}^{2} \cdot \mathrm{ha}^{-1}\right) \times\left(24 \mathrm{~h} \cdot \mathrm{d}^{-1}\right) \\
& \times\left(365 \mathrm{~d} \cdot \mathrm{yr}^{-1}\right) \times\left(14 \mathrm{~g} \cdot \mathrm{mol}^{-1}\right) \times\left(0.001 \mathrm{~kg} \cdot \mathrm{g}^{-1}\right)=9.96 \mathrm{~kg} \cdot \mathrm{N} \cdot \mathrm{ha}^{-1} \cdot \mathrm{yr}^{-1}
\end{aligned}
$$

or $9.96-10.7 \mathrm{~kg} \cdot \mathrm{N} \cdot \mathrm{ha}^{-1} \cdot \mathrm{yr}^{-1}$, and similar to $9.88 \mathrm{~kg} \cdot \mathrm{N} \cdot \mathrm{ha}^{-1} \cdot \mathrm{yr}^{-1}$ estimated by co-workers (in reference [33]'s Table 3);

2) Likewise, West River median area-weighted $\mathrm{N}_{2}$ flux range 1100 - 1149 $\mu \mathrm{mol} \cdot \mathrm{N} \cdot \mathrm{m}^{-2} \cdot \mathrm{h}^{-1}$ (Table 6) or $13.49-14.09 \mathrm{~kg} \cdot \mathrm{N} \cdot \mathrm{ha}^{-1} \cdot \mathrm{yr}^{-1}$, is similar to $14.06 \mathrm{esti}-$ mated by co-workers (in Reference [33]'s Table 3).

\subsection{Case Study 5: Comparison between Denitrification Rates (Median and Interquartile) Measured on the Elbe River with Rates Predicted by Our Model}

Elbe River denitrification rates were obtained by measuring dissolved $\mathrm{N}_{2}$ "super-saturation" (measured dissolved $\mathrm{N}_{2}$ minus equilibrium dissolved $\mathrm{N}_{2}$ ) and 
Table 6. Source data (from Reference [33]'s Figure 3) are shown in bold font. Data from tributary sites' (W1, W2 and W3) were not used here.

\begin{tabular}{|c|c|c|c|c|c|c|}
\hline \multirow[b]{2}{*}{ Site } & \multirow{2}{*}{$\begin{array}{c}\left(\mathrm{NH}_{4}-\mathrm{N}\right)+ \\
\left(\mathrm{NO}_{2}-\mathrm{N}\right)+ \\
\left(\mathrm{NO}_{3}-\mathrm{N}\right) \\
\left(\mu \mathrm{mol} \cdot \mathrm{N} \cdot \mathrm{l}^{-1}\right) \\
(\mathrm{A})\end{array}$} & \multirow[b]{2}{*}{$\begin{array}{c}\Delta \mathrm{N}_{2} \\
\left(\mu \mathrm{mol} \cdot \mathrm{N} \cdot \mathrm{l}^{-1}\right)^{\mathrm{a}} \\
\text { (B) }\end{array}$} & \multirow[b]{2}{*}{$A+B$} & \multicolumn{3}{|c|}{ Denitrification $\left(\mu \mathrm{mol} \cdot \mathrm{N} \cdot \mathrm{m}^{-2} \cdot \mathrm{h}^{-1}\right)$} \\
\hline & & & & $\begin{array}{l}\text { Diffusive } \\
\text { estimated } \\
\text { by us }^{\mathrm{b}} \\
\text { (C) }\end{array}$ & $\begin{array}{c}\text { Ebullitive } \\
\text { estimated by } \\
\text { us }^{\mathrm{c}} \\
\text { (D) }\end{array}$ & $\mathrm{C}+\mathrm{D}$ \\
\hline \multicolumn{7}{|c|}{ North River } \\
\hline N1 & 670 & 160 & 830 & $505-526$ & 1341 & $1847-1867$ \\
\hline N2 & 630 & 163 & 793 & $499-522$ & 1281 & $1780-1802$ \\
\hline N3 & 538 & 130 & 668 & $473-504$ & 1076 & $1549-1580$ \\
\hline N4 & 600 & 110 & 710 & $483-511$ & 1145 & $1627-1655$ \\
\hline N5 & 268 & 64 & 332 & $356-415$ & 526 & $882-941$ \\
\hline N6 & 200 & 52 & 252 & $308-373$ & 395 & $703-768$ \\
\hline N7 & 235 & 50 & 285 & $330-392$ & 449 & $778-841$ \\
\hline N8 & 223 & 62 & 285 & $330-392$ & 449 & $778-841$ \\
\hline N9 & 240 & 44 & 284 & $329-392$ & 447 & $776-839$ \\
\hline N10 & 248 & 46 & 294 & $335-397$ & 463 & $799-860$ \\
\hline N11 & 238 & 54 & 292 & $334-396$ & 460 & $794-856$ \\
\hline N12 & 250 & 50 & 300 & $339-400$ & 473 & $812-873$ \\
\hline N13 & 245 & 48 & 293 & $335-396$ & 462 & $796-858$ \\
\hline N14 & 300 & 50 & 350 & $366-423$ & 555 & $921-978$ \\
\hline N15 & 295 & 54 & 349 & $365-422$ & 554 & $919-976$ \\
\hline \multicolumn{6}{|c|}{ North River median emission range } & $812-873$ \\
\hline \multicolumn{7}{|c|}{ West River } \\
\hline W4 & 670 & 72 & 742 & $489-515$ & 1187 & $1686-1712$ \\
\hline W5 & 725 & 76 & 801 & $500-523$ & 1286 & $1794-1816$ \\
\hline W6 & 180 & 86 & 266 & $318-382$ & 395 & $735-799$ \\
\hline w7 & 190 & 74 & 264 & $316-381$ & 404 & $731-795$ \\
\hline W8 & 250 & 88 & 338 & $360-418$ & 526 & $895-953$ \\
\hline W9 & 140 & 72 & 212 & $279-346$ & 300 & $608-675$ \\
\hline W10 & 243 & 86 & 329 & $355-414$ & 504 & $876-934$ \\
\hline W11 & 350 & 100 & 450 & $409-457$ & 701 & $1128-1176$ \\
\hline $\mathrm{W} 12$ & 340 & 96 & 436 & $404-453$ & 667 & $1100-1149$ \\
\hline W13 & 340 & 100 & 440 & $405-454$ & 671 & $1108-1157$ \\
\hline W14 & 400 & 106 & 506 & $429-472$ & 785 & $1240-1283$ \\
\hline \multicolumn{6}{|c|}{ West River median emission range } & $1100-1149$ \\
\hline
\end{tabular}

${ }^{\mathrm{a}}$ Dissolved $\mathrm{N}_{2}$, in excess of environmental (background) dissolved $\mathrm{N}_{2} \cdot{ }^{\mathrm{b}}[(700 \times(\mathrm{A}+\mathrm{B})) /(320+(\mathrm{A}+\mathrm{B}))]$ and $[(640 \times(\mathrm{A}+\mathrm{B})) /(180+(\mathrm{A}+\mathrm{B}))]$, see Table 3. ${ }^{\mathrm{c}}[(1.638 \times(\mathrm{A}+\mathrm{B}))-18.11]$, see Table 3. 
using varying reaeration-rate coefficient gas exchange equations [34]. Comparison shows:

1) The decreasing $\mathrm{N}_{2}$ emission tendency from upstream (Reach A) to downstream (Reach C) is observed in both works, here and [34], (Table 7).

2) Median (and interquartile) total $N$ in the Elbe River water between years 2011 and 2012 was $5.0(4.0 ; 6.1) \mathrm{mg} \mathrm{N} / \mathrm{l}$ based on 863 measurements (http://www.fgg-elbe.de) performed on the about $105 \mathrm{~km}^{2}$ studied river area. Using this data and the model here proposed we estimate a nitrogen removal of $12,910(11,038 ; 14,728) \mathrm{t} \cdot \mathrm{N} \cdot \mathrm{yr}^{-1}$. These rates are compatible with the $\sim 10,000$ $\mathrm{t} \cdot \mathrm{N} \cdot \mathrm{yr}^{-1}$ extrapolated annual estimate, based on annual temperature changes [34].

\section{Discussion}

To the best of our knowledge, this is the first work to report $\mathrm{N}_{2} \mathrm{aec}$ in ebullitive emission samples using $\mathrm{Ar}$ as a tracer of environmental $\mathrm{N}_{2}$. Furthermore, this approach can be used to quantify ebullitive $\mathrm{N}_{2}$ aec in bubbles sampled elsewhere (Table 8 and Table 9).

Using a different analytical approach-headspace equilibration-gaseous $\mathrm{N}_{2}: \mathrm{Ar}$ in the collected bubbles at sites from South Platte River within $81 \mathrm{~km}$ of Denver (Colorado, USA) were quantified [35]. The collected bubbles were injected into a vial containing $40 \mathrm{ml}$ of $\mathrm{N}_{2}$-saturated water and shaken for 1 minute. Dissolved $\mathrm{N}_{2}$ and Ar concentrations in the liquid fraction, measured via MIMS [36] [37], were used to back-calculate gas concentrations in the collected bubbles. The $\mathrm{N}_{2}$ aec-possibly denitrified $\mathrm{N}_{2}$ in fact-in those bubbles ranged from $0 \%$ to $13.9 \%$ (Table 9).

The rate of increase of total $\mathrm{N}_{2}$ aec emission weakens with dissolved $\mathrm{N}$ concentration: from $3 \mu \mathrm{mol} \mathrm{N}{ }_{2} \mathrm{aec}-\mathrm{N} \cdot \mathrm{m}^{-2} \cdot \mathrm{h}^{-1} / \mu \mathrm{mol} \cdot \mathrm{N} \cdot \mathrm{l}^{-1}$ (Table 3) to 1.797 (Table 3) to 0.4315 (Table 3). The denitrification (a source of $\mathrm{N}_{2}$ aec) model from co-workers also points to a decrease in denitrification capability of streams with increasing nitrate loads: "higher loading rates stimulate $\mathrm{NO}_{3}^{-}$uptake and denitrification, but yield an associate disproportionate increase in downstream $\mathrm{NO}_{3}^{-}$ export to receiving waters" [38].

While it is possible that the $\mathrm{N}_{2}$ gas in bubbles is due to "excess air" from groundwater recharge, the fact that the ratio N:Ar in this "excess air" is close to that in the atmosphere [39] excludes the $\mathrm{N}_{2}$ of this source to be accounted as denitrified $\mathrm{N}_{2}$. This suggests denitrification is the source of excess $\mathrm{N}_{2}$ in the bubbles here reported.

Possible causes for negative emission rates are: $\mathrm{N}$ consumption, $\mathrm{N}$ fixation in excess of production, excess $\mathrm{O}_{2}$ (possibly from photosynthesis), and/or Ar gas seeping into the bubble faster than $\mathrm{O}_{2}$ or $\mathrm{N}_{2}$. If the cause was $\mathrm{N}$ consumption or $\mathrm{N}$ fixation in excess of production, then inclusion of the negative rates in median calculation would yield net $\mathrm{N}_{2}$ "production". However, median emission results for each one of the eleven surveys would not be impacted by this inclusion except for São Marcos' River June 2011 survey 2/3 (median would be 
Table 7. Dissolved $\mathrm{N}$ and $\mathrm{N}_{2}$ emissions from Elbe River (source data [34] in bold).

\begin{tabular}{ccccc}
\hline Campaign-Reach & Total N $(\mathrm{mg} / \mathrm{l})^{\mathrm{a}}$ & $\mathrm{n}^{\mathrm{b}}$ & $\begin{array}{c}\text { Median rate and } \\
\text { ranges estimated here } \\
\left(\mathrm{mg} \cdot \mathrm{N} \cdot \mathrm{m}^{-2} \cdot \mathrm{h}^{-1}\right)\end{array}$ & $\begin{array}{c}\text { Median rate and ranges } \\
\text { estimated by Ritz et al. } \\
{[34]\left(\mathrm{mg} \cdot \mathrm{N} \cdot \mathrm{m}^{-2} \cdot \mathrm{h}^{-1}\right)}\end{array}$ \\
\hline Summer 2011-A & $5.8(5.2 ; 6.1)$ & 22 & $15(14 ; 16)$ & $14(11 ; 22)$ \\
Summer 2011-B & $4.8(4.5 ; 4.9)$ & 6 & $14(13 ; 14)$ & $25(16 ; 34)$ \\
Summer 2011-C & $2.6(2.6 ; 2.9)$ & 3 & $8(8 ; 9)$ & $6(2 ; 6)$ \\
Spring 2012-A & $4.9(4.3 ; 5.2)$ & 18 & $14(13 ; 14)$ & $21(18 ; 33)$ \\
Spring 2012-B & $4.3(4.1 ; 4.3)$ & 5 & $13(12 ; 13)$ & $11(1 ; 12)$ \\
Spring 2012-C & $2.8(2.6 ; 2.9)$ & 3 & $8(8 ; 9)$ & $10(-1 ; 13)$
\end{tabular}

ahttp://www.fgg-elbe.de. ${ }^{b}$ Quantity of measurements. ${ }^{~}$ Total $\mathrm{N}$ is multiplied by $71.4(1 \mathrm{mg} \mathrm{N}=71.4 \mu \mathrm{mol}$ $\mathrm{N})$. The appropriate equation is selected from "Total emission" column in Table 3 . The result is divided by 71.4 .

Table 8. Columns "Site", " $\% \mathrm{O}_{2}+\mathrm{Ar}$ " and " $\% \mathrm{~N}_{2}$ " are from reference [27]. There it is stated that $5 \%$ of the $\mathrm{O}_{2}+\mathrm{Ar}$ mixture is $\mathrm{Ar}$ (calculated in the "\% Ar" column). Assuming environmental concentrations $78 \% \mathrm{~N}_{2}$ and $0.93 \% \mathrm{Ar}$, we used $\mathrm{Ar}$ as a tracer of environmental $\mathrm{N}_{2}$ to calculate the concentration (\%) of $\mathrm{N}_{2}$ aec (possibly denitrified $\mathrm{N}_{2}$ ) in bubbles (results in "\% $\mathrm{N}_{2}$ aec-N" column). Example of calculation (using data from line 1, Site DC): $5.40=7.5-\left(0.025 \% \mathrm{Ar} \times 78 \% \mathrm{~N}_{2} / 0.93 \% \mathrm{Ar}\right)$. We assumed negative values indicated zero $\mathrm{N}_{2}$ aec in bubbles (see Table A1 header). Median (24.12) was not changed by including negative (rather than zero) value observed in site CLB.

\begin{tabular}{|c|c|c|c|c|}
\hline Site & $\%\left(\mathrm{O}_{2}+\mathrm{Ar}\right)$ & $\% \mathrm{~N}_{2}$ & $\% \mathrm{Ar}^{\dagger}$ & $\% \mathrm{~N}_{2}$ aec-N \\
\hline $\mathrm{DC}$ & 0.5 & 7.5 & 0.025 & 5.40 \\
\hline BB & 1.2 & 36.4 & 0.06 & 31.37 \\
\hline $\mathrm{HC}$ & 0.3 & 11.1 & 0.015 & 9.84 \\
\hline CLB & 0.9 & 3.4 & 0.045 & $-0.37(0)$ \\
\hline UF & 1.1 & 29.5 & 0.055 & 24.89 \\
\hline GI & 1.3 & 24.4 & 0.065 & 18.95 \\
\hline$S$ & 1.2 & 12 & 0.06 & 6.97 \\
\hline F & 1.3 & 28.8 & 0.065 & 23.35 \\
\hline $\mathrm{Br}$ & 1 & 42.7 & 0.05 & 38.51 \\
\hline LF & 1.4 & 49.8 & 0.07 & 43.93 \\
\hline $\mathrm{HL}$ & 0.6 & 19.4 & 0.03 & 16.88 \\
\hline Pelt. & 2.1 & 64.4 & 0.105 & 55.59 \\
\hline Spar. & 2.4 & 84.5 & 0.12 & 74.44 \\
\hline $\mathrm{DFC}$ & 1.2 & 52.1 & 0.06 & 47.07 \\
\hline \multicolumn{4}{|c|}{ Median } & $24.12(24.12)$ \\
\hline
\end{tabular}

${ }^{\dagger} 5 \%$ of $\left(\mathrm{O}_{2}+\mathrm{Ar}\right)$.

-3.27 $\mathrm{mg} \cdot \mathrm{N}_{2}-\mathrm{N} \cdot \mathrm{m}^{-2} \cdot \mathrm{d}^{-1}$, rather than zero) as shown in Table A1, indicating that in the six rivers here studied $\mathrm{N}$ production would exceed $\mathrm{N}$ fixation. Excess $\mathrm{N}_{2}$ was also found in most bubble samples collected in the White Oak River estuary USA (Table 8) and South Platte River USA (Table 9). 
Table 9. Columns "Site", "Distance" and "Gaseous $\mathrm{N}_{2}$ :Ar" were copied form Reference [35]. Sites are situated on South Platte River downstream from the point of discharge from the largest wastewater treatment plant from Denver, serving 1.3 million people. Concentration of $\mathrm{N}_{2}$ aec was calculated using environmental ratio $78 \% \mathrm{~N}_{2}: 0.93 \%$ Ar. Calculation example for the South Plate site: $(79.93 \times 0.93)-78=-3.67(0)$. Median $(7.51)$ was not changed by inclusion of negative value observed in South Platte site.

\begin{tabular}{cccc}
\hline Site & $\begin{array}{c}\text { Distance from wastewater discharge } \\
(\text { river } \mathrm{km})\end{array}$ & Gaseous $\mathrm{N}_{2}: \mathrm{Ar}$ & $\begin{array}{c}\mathrm{N}_{2} \text { aec-N in } \\
\text { bubbles (\%) }\end{array}$ \\
\hline South Platte & -18 & $\mathbf{7 9 . 9 3}$ & $-3.67(0)$ \\
& +5 & 95.28 & 10.6 \\
Site A & +10 & $\mathbf{9 7 . 0 7}$ & 12.3 \\
& $+\mathbf{+ 1 6}$ & $\mathbf{9 7 . 5 1}$ & 12.7 \\
Site B & $\mathbf{+ 2 4}$ & $\mathbf{9 8 . 8 6}$ & 13.9 \\
Site C & $\mathbf{+ 4 4}$ & $\mathbf{8 8 . 6 2}$ & 4.42 \\
& $\mathbf{+ 5 2}$ & $\mathbf{8 7 . 6 4}$ & 3.51 \\
Site D & $\mathbf{+ 8 1}$ & $\mathbf{8 7 . 5 5}$ & 3.42 \\
& Median & & $7.51(7.51)$ \\
\hline
\end{tabular}

Synthetically stirred bubbles from bubble-enriched sediment sites [27] showed higher median concentration (24.1\% $\mathrm{N}_{2}$ aec- $\mathrm{N}, \mathrm{n}=14$, Table 8) of $\mathrm{N}_{2} \mathrm{aec}$ than ours $\left(5.75 \% \mathrm{~N}_{2}\right.$ aec-N, $\mathrm{n}=112$, data from Table A1) and, than the median (7.51\% $\mathrm{N}_{2}$ aec-N, $\mathrm{n}=8$, Table 9) for naturally emerging bubbles from a high nutrient segment of South Platte River. The relatively small median (5.75\%) obtained here could be due to partial bubble dissolution during the diel harvest, or greater variety of sampled aquatic environments.

The significant rates of ebullitive gas water-air emission from the heavily urbanized stretches of Pinheiros and Tietê rivers here observed support the finding that urban streams and rivers should be included in river nitrogen cycling models [40].

Bubble occurrence in the eyes and inwards of fish [41] is a condition known as "gas bubble disease" also referred to as "gas bubble trauma". This can possibly be explained by super saturation levels of dissolved $\mathrm{N}$ concentration $\left(>\sim 250 \mu \mathrm{mol} \cdot \mathrm{l}^{-1}\right)$ because this favors excessive bubble formation while promoting the ebullitive escape discussed here.

\section{Conclusions}

In waters with small concentrations of dissolved $\mathrm{N}\left(<10 \mu \mathrm{mol} \cdot \mathrm{N} \cdot \mathrm{l}^{-1}\right)$ ebullitive $\mathrm{N}_{2}$ aec water-air emissions are practically insignificant and diffusive $\mathrm{N}_{2}$ aec emissions predominate. Ebullitive and diffusive $\mathrm{N}_{2}$ aec emissions increase with dissolved $\mathrm{N}$ concentration but diffusive $\mathrm{N}_{2}$ aec emissions saturate at $\sim 700$ $\mu \mathrm{mol} \cdot \mathrm{N} \cdot \mathrm{m}^{-2} \cdot \mathrm{h}^{-1}$ in waters with $>\sim 1000 \mu \mathrm{mol} \cdot \mathrm{N} \cdot \mathrm{l}^{-1}$, while ebullitive continue to grow.

While the chromatographic analyses of $\mathrm{N}_{2}$ and $\mathrm{O}_{2}$ (although $\mathrm{CH}_{4}$ and $\mathrm{CO}_{2}$ 
were the main gases of interest at the time) were carefully done, the Ar concentration in the ebullitive emission sample hinges on a statement, i.e. that $5 \%$ of the " $\mathrm{O}_{2}$ " peak is Ar. Here, there is margin for refinement because the Ar concentration in each ebullitive emission sample can easily be done chromatographically, using $\mathrm{O}_{2}$ as a carrier gas, with the precision required for this work.

As dissolved $\mathrm{N}$ is a predictor of $\mathrm{N}_{2}$ aec emission, denitrified $\mathrm{N}_{2}$ emission models would benefit from data of simultaneous measurements of total dissolved $\mathrm{N}$ concentration $\left(\mathrm{NH}_{4}-\mathrm{N}+\mathrm{NO}_{3}-\mathrm{N}+\mathrm{NO}_{2}-\mathrm{N}+\mathrm{N}_{2}-\mathrm{N}\right)$ and denitrified $\mathrm{N}_{2}$ ebullitive and diffusive emission (rather than not measuring total dissolved $\mathrm{N}$ along with the ebullitive and diffusive measurements).

Our $\mathrm{N}_{2}$ aec emission model predicted 13\% - 15\% $(63-50=13$ and $65-50=$ 15) more ebullitive $\mathrm{N}$ losses than the actual "missing N", in Case Study 1. Causes for this overestimation could probably be understood with more bubble emission measurements in other river systems, considering local variability such as slope, sediment types, $\mathrm{N}$ inputs and biochemistry. In Case Study 2, the nitrogen-removal range predicted by our model tends to be within the measured range by MIMS, except when nitrate concentrations are highest (840 and 1096 $\mu \mathrm{mol} \mathrm{N} / \mathrm{l}$ ) then, our model overestimates the measured nitrogen-removal rates. This could be due to local conditions and/or underestimation by MIMS of the ebullitive nitrogen-removal pathway. In Case Studies 3, 4 and 5 the measured values by co-workers are predicted relatively well by our model.

\section{Acknowledgements}

We thank Professor John Prausnitz for acknowledging that our explanation for the observed bubble behavior is reasonable. We thank field survey colleagues and boatmen from the Carbon Budget Brazil projects; also F. S. David and W. C. Valenti for priming our interest in the nitrogen cycle and B. Matvienko for the insightful discussions on, given the amassed data how can we quantify denitrified $\mathrm{N}_{2}$ bubble emission; D. Sikar and B. Matvienko for helping to survey the polluted rivers of the São Paulo city metropolitan area; I. and P. Matvienko-Sikar, L.R.C. Ribeiro and R. L. North for their comments; and ten anonymous reviews of earlier versions of this work. We thank Trimmer et al. 2012 [42] for leading us to discover where the excess nitrogen we found in bubbles, was being missed. For permission to use results of $\mathrm{O}_{2}, \mathrm{~N}_{2}, \mathrm{CH}_{4}$ and $\mathrm{CO}_{2}$ in bubble samples, we thank: Prefeitura de São Paulo/Secretaria do Verde e Meio Ambiente, ANTP-Associação Nacional de Transportes Públicos (data from 2 rivers in the São Paulo city metropolitan area), Ministry of Mines and Energy-MME and Eletronorte (data from the other 4 rivers). "Take note of the $\mathrm{O}_{2}$ and $\mathrm{N}_{2}$ concentrations in bubbles, you might discover something” Professor Bohdan Matvienko $\left({ }^{\star} 1933 \dagger 2013\right)$, to whom we dedicate this work.

\section{Conflicts of Interest}

The authors declare no conflict of interest, financial or otherwise. 


\section{References}

[1] Seitzinger, S., Harrison, J.A., Böhlke, J.K., et al. (2006) Denitrification across Landscapes and Waterscapes: A Synthesis. Ecological Applications, 16, 2064-2090. https://doi.org/10.1890/1051-0761(2006)016[2064:DALAWA]2.0.CO;2

[2] Baulch, H.M., Dillon, P.J., Maranger, R., et al. (2011) Diffusive and Ebullitive Transport of Methane and Nitrous Oxide from Streams: Are Bubble-Mediated Fluxes Important? Journal of Geophysical Research, 116, Article ID: G04028. https://doi.org/10.1029/2011JG001656

[3] Duchemin, E., Lucotte, M. and Canuel, R. (1999) Comparison of Static Chamber and Thin Boundary Layer Equation Methods for Measuring Greenhouse Gas Emissions from Large Water Bodies. Environmental Science and Technology, 33, 350-357. https://doi.org/10.1021/es9800840

[4] Groffman, P.M. (2012) Terrestrial Denitrification: Challenges and Opportunities. Ecological Processes, 1, 1-11. https://doi.org/10.1186/2192-1709-1-11

[5] Matvienko, B., Sikar, E., Rosa, L.P., et al. (2000) Gas Release from a Reservoir in the Filling Stage. Verhandlungen der Internationalen Vereinigung für Theoretische und Angewandte Limnologie, 27, 1415-1419. https://doi.org/10.1080/03680770.1998.11901469

[6] Goldenfum, J.A. (2018) GHG Measurement Guidelines for Freshwater Reservoirs. Derived from: The UNESCO/IHA Greenhouse Gas Emissions from Freshwater Reservoirs Research Project; International Hydropower Association. https://www.hydropower.org/sites/default/files/publications-docs/GHG\%20Measur ement\%20Guidelines\%20for\%20Freshwater\%20Reservoirs.pdf

[7] Santos, M.A., Rosa, L.P., Sikar, B., et al. (2006) Gross Greenhouse Gas Fluxes from Hydro-Power Reservoir Compared to Thermo-Power Plants. Energy Policy, 34, 481-488. https://doi.org/10.1016/j.enpol.2004.06.015

[8] Merck Research Laboratories (2006) Merck Index: An Encyclopedia of Chemicals, Drugs, and Biologicals. Merck Research Laboratories, Whitehouse Station, NJ.

[9] NOAA (Earth System Research Laboratory Global Monitoring Division) (2018) The NOAA Annual Greenhouse Gas Index (AGGI). https://www.esrl.noaa.gov/gmd/aggi/aggi.html

[10] Weiss, R.F. (1970) The Solubility of Nitrogen, Oxygen and Argon in Water and Seawater. Deep-Sea Research, 17, 721-735.

https://doi.org/10.1016/0011-7471(70)90037-9

[11] Fox, R.J., Fisher, T.R., Gustafson, A.B., et al. (2014) Searching for the Missing Nitrogen: Biogenic Nitrogen Gases in Groundwater and Streams. Journal of Agricultural Science, 152, S96-S106. https://doi.org/10.1017/S0021859614000070

[12] Böhlke, J.K., Antweiler, R.C., Harvey, J.W., et al. (2009) Multi-Scale Measurements and Modeling of Denitrification in Streams with Varying Flow and Nitrate Concentration in the Upper Mississippi River Basin, USA. Biogeochemistry, 93, 117-141. https://doi.org/10.1007/s10533-008-9282-8

[13] Davidson, E.A. and Seitzinger, S. (2006) The Enigma of Progress in Denitrification Research. Ecological Applications, 16, 2057-2063. https://doi.org/10.1890/1051-0761(2006)016[2057:TEOPID]2.0.CO;2

[14] Schlesinger, W.H. (2009) On the Fate of Anthropogenic Nitrogen. Procedures National Academy of Sciences, 106, 203-208. https://doi.org/10.1073/pnas.0810193105

[15] Howarth, R.W., Billen, G., Swaney, D., et al. (1996) Regional Nitrogen Budgets and Riverine N \& P Fluxes for the Drainages to the North Atlantic Ocean: Natural and 
Human Influences. Biogeochemistry, 35, 75-139. https://doi.org/10.1007/BF02179825

[16] Jordan, T.E. and Weller, D.E. (1996) Human Contributions to Terrestrial Nitrogen Flux. BioScience, 46, 655-664. https://doi.org/10.2307/1312895

[17] Williams, M.R., Filoso, S., Martinelli, L.A., et al. (2001) Precipitation and River Water Chemistry of the Piracicaba River Basin, Southeast Brazil. Journal of Environmental Quality, 30, 967-981. https://doi.org/10.2134/jeq2001.303967x

[18] Boyer, E.W., Goodale, C.L., Jaworski, N.A., et al. (2002) Anthropogenic Nitrogen Sources and Relationships to Riverine Nitrogen Export in the Northeastern USA. Biogeochemistry, 57-58, 137-169. https://doi.org/10.1023/A:1015709302073

[19] Van Breemen, N., Boyer, E.W., Goodale, C.L., et al. (2002) Where Did All the Nitrogen Go? Fate of Nitrogen Inputs to Large Watersheds in the Northeastern USA. Biogeochemistry, 57, 267-293.

[20] Filoso, S., Martinelli, L.A., Williams, M.R., et al. (2003) Land Use and Nitrogen Export in the Piracicaba River Basin, Southeast Brazil. Biogeochemistry, 65, 275-294. https://doi.org/10.1023/A:1026259929269

[21] Schaefer, S.C. and Alber, M. (2007) Temperature Controls a Latitudinal Gradient in the Proportion of Watershed Nitrogen Exported to Coastal Ecosystems. Biogeochemistry, 85, 333-346. https://doi.org/10.1007/s10533-007-9144-9

[22] Gardner, J.R., Fisher, T.R., Jordan, T.E., et al. (2016) Balancing Watershed Nitrogen Budgets: Accounting for Biogenic Gases in Streams. Biogeochemistry, 127, 231-253. https://doi.org/10.1007/s10533-015-0177-1

[23] CETESB State of São Paulo's Environmental Agency (2012) Reports of Surface Waters' Quality in the Brazilian State of São Paulo. (In Portuguese) https://cetesb.sp.gov.br/

[24] IIEGA International Institute of Ecology and Environmental Management Report II (2009) Surface Water Quality of Arara, Paquiçamba and Juruna Native Land of the Belo Monte Hydroelectric Facility Area. (In Portuguese)

http://philip.inpa.gov.br/publ_livres/Dossie/BM/DocsOf/EIA-09/Vol\%2035/TOMO \%201/Anexos/Anexo\%202.2.1-1/Texto/Rel2_jan09_IIEGA.pdf

[25] Santos, M.A., Matvienko, B., Rosa, L.P., et al. (2005) Gross Greenhouse Gas Emissions from Brazilian Hydro Reservoirs. In: Tremblay, A., Varfalvy, L., Roehm, C. and Garneau, M., Eds., Greenhouse Gas Emissions-Fluxes and Processes, Springer, Berlin, 267-291.

[26] Preto, B.L., Henares, M.N.P., Kimpara, J.M., et al. (2015) Greenhouse Emissions from Aquaculture. Global Aquaculture Advocate.

https://www.researchgate.net/profile/Wagner_Valenti2/publication/281101561_Green house_emissions_from_aquaculture/links/55d4e4ee08ae1e651663715c/Greenhouse-e missions-from-aquaculture.pdf

[27] Chanton, J.P., Martens, C.S. and Kelley, C.A. (1989) Gas Transport from Methane-Saturated, Tidal Freshwater and Wetland Sediments. Limnology and Oceanography, 34, 807-819. https://doi.org/10.4319/1o.1989.34.5.0807

[28] Epstein, P.S. and Plesset, M.S. (1950) On the Stability of Gas Bubbles in Liquid-Gas Solutions. The Journal of Chemical Physics, 18, 1505-1509.

https://doi.org/10.1063/1.1747520

[29] Harper, L.A., Sharpe, R.R., Parkin, T.B., et al. (2004) Nitrogen Cycling through Swine Production Systems: Ammonia, Dinitrogen and Nitrous Oxide Emissions. Journal of Environmental Quality, 33, 1189-1201. https://doi.org/10.2134/jeq2004.1189 
[30] Smith, L.K., Voytek, M.A., Böhlke, J.K., et al. (2006) Denitrification in Nitrate-Rich Streams: Application of $\mathrm{N}_{2}$ :Ar and ${ }^{15} \mathrm{~N}$-Tracer Methods in Intact Cores. Ecological Applications, 16, 2191-2207. https://doi.org/10.1890/1051-0761(2006)016[2191:DINSAO]2.0.CO;2

[31] Chen, N., Chen, Z., Wu, Y., et al. (2014) Understanding Gaseous Nitrogen Removal through Direct Measurement of Dissolved $\mathrm{N}_{2}$ and $\mathrm{N}_{2} \mathrm{O}$ in a Subtropical River-Reservoir System. Ecological Engineering, 70, 56-67. https://doi.org/10.1016/j.ecoleng.2014.04.017

[32] Sikar, E., Matvienko, B., Santos, M.A., et al. (2012) Silicon as a Permanent-Carbon Sedimentation Tracer. Inland Waters, 2, 119-128. https://doi.org/10.5268/IW-2.3.465

[33] Chen, N., Wu, J., Chen, Z., et al. (2014) Spatial-Temporal Variation of Dissolved $\mathrm{N}_{2}$ and Denitrification in an Agricultural River Network, Southeast China. Agriculture, Ecosystems and Environment, 189, 1-10. https://doi.org/10.1016/j.agee.2014.03.004

[34] Ritz, S., Dähnke, K. and Fischer, H. (2018) Open-Channel Measurement of Denitrification in a Large Lowland River. Aquatic Sciences, 80, 11.

https://doi.org/10.1007/s00027-017-0560-1

[35] Higgins, T.M., McCutchan Jr., J.H. and Lewis Jr., W.M. (2008) Nitrogen Ebullition in a Colorado Plains River. Biogeochemistry, 89, 367-377. https://doi.org/10.1007/s10533-008-9225-4

[36] Kana, T.M., Darkangelo, C., Hunt, M.D., et al. (1994) Membrane Inlet Mass Spectrometer for Rapid High-Precision Determination of $\mathrm{N}_{2}, \mathrm{O}_{2}$, and Ar in Environmental Water Samples. Analytical Chemistry, 66, 4166-4170.

https://doi.org/10.1021/ac00095a009

[37] McCutchan, J.H., Saunders, J.F.I., Pribyl, A.L., et al. (2003) Open-Channel Estimation of Denitrification. Limnology and Oceanography: Methods, 1, 74-81. https://doi.org/10.4319/lom.2003.1.74

[38] Mulholland, P.J., Helton, A.M., Poole, G.C., et al. (2008) Stream Denitrification across Biomes and Its Response to Anthropogenic Nitrate Loading. Nature, 452, 202-205. https://doi.org/10.1038/nature06686

[39] Heaton, T.H.E. and Vogel, J.C. (1981) "Excess Air" in Groundwater. Journal of Hydrology, 50, 201-216. https://doi.org/10.1016/0022-1694(81)90070-6

[40] Helton, A.M., Poole, G.C., Meyer, J.L., et al. (2011) Thinking outside the Channel: Modeling Nitrogen Cycling in Networked River Ecosystems. Frontiers in Ecology and the Environment, 9, 229-238. https://doi.org/10.1890/080211

[41] McGrath, K.E., Dawley, E.M. and Geist, D.R. (2006) Total Dissolved Gas Effects on Fishes of the Lower Columbia River. Final Report, Prepared for the U.S. Army Corps of Engineers Portland District, Portland, Oregon under a Related Services Agreement with the U.S. Department of Energy Contract DE-AC05-76RL01830.

[42] Trimmer, M., Grey, J., Heppell, C.M., et al. (2012) River Bed Carbon and Nitrogen Cycling: State of Play and Some New Directions. Science of the Total Environment, 434, 143-158. https://doi.org/10.1016/j.scitotenv.2011.10.074 


\section{Appendix}

Table A1 Each of the six rivers' surveyed sites. ${ }^{\text {aSite }}$ depth. ${ }^{\text {bVolume of collected }}$ bubble gas. 'Quantity of $70 \mathrm{~cm}$ diameter funnels at sampled site. ${ }^{\mathrm{d}}$ Sampling time interval. ${ }^{\mathrm{e} S u r f a c e}$ water temperature, at $\sim 10 \mathrm{~cm}$ depth. ${ }^{\mathrm{f}}$ Atmospheric pressure in field laboratory. ${ }^{\mathrm{g}} \mathrm{O}_{2}+\mathrm{Ar}, \mathrm{N}_{2}, \mathrm{CO}_{2}$ and $\mathrm{CH}_{4}$ concentrations in bubble gas samples. ${ }^{\text {h}}$ See $2.5 \mathrm{~N}_{2}$ aec bubble-emission calculation method (main text). ${ }^{\text {EExcluded, river }}$ flow probably tilted the funnels and air entered bubble-gas vial. 'No existing data. ${ }^{k}$ Median temperature; temperature probe had faulty contact. ${ }^{1}$ Underestimated volume due to bubble-gas overflow. ${ }^{\mathrm{m}}$ Not analyzed. ${ }^{\mathrm{n}}$ First and third quartiles are the nonparametric statistics' numerical equivalents to the normal distribution's two values in between which the central $50 \%$ of the area under the normal curve lies. First and third quartiles were calculated assuming: ebullitive $\mathrm{N}_{2}$ aec emission rate histograms of polluted rivers display normal distributions; \pm 1 standard deviation from the mean were 293 and 405 (Tietê River) and 858 and 1011 (Pinheiros River). ${ }^{\dagger}$ Negative emissions, and results of calculations using negative emissions, are italicized. This parallel calculation was done for the following reason: if the assumption that negative $\mathrm{N}_{2}$ emission rates indicate $\mathrm{N}$ fixation is true, then their inclusion in median calculation would yield either net $\mathrm{N}_{2}$ production (positive median) or net uptake of $\mathrm{N}$ i.e. consumption (negative median). 
Table A1. (a) Xingu River; (b) Tocantins River; (c) Madeira River; (d) São Marcos River; (e) Tietê River; (f) Pinheiros River.

(a)

\begin{tabular}{|c|c|c|c|c|c|c|c|c|c|c|c|c|}
\hline \multicolumn{2}{|c|}{ Site } & \multirow{2}{*}{$\begin{array}{l}\mathrm{SD}^{\mathrm{a}} \\
(\mathrm{m})\end{array}$} & \multirow{2}{*}{$\begin{array}{c}\mathrm{VCBG}^{\mathrm{b}} \\
(\mathrm{ml})\end{array}$} & \multirow[b]{2}{*}{$\mathrm{QF}^{c}$} & \multirow{2}{*}{$\begin{array}{l}\text { STI }^{\mathrm{d}} \\
(\mathrm{h})\end{array}$} & \multirow{2}{*}{$\begin{array}{l}\mathrm{WT}^{\mathrm{e}} \\
(\mathrm{K})\end{array}$} & \multirow{2}{*}{$\begin{array}{c}\mathrm{AP}^{\mathrm{f}} \\
(\mathrm{mm} \mathrm{Hg})\end{array}$} & \multirow{2}{*}{$\begin{array}{c}\mathrm{O}_{2}+\mathrm{Ar}^{\mathrm{g}} \\
(\%)\end{array}$} & \multirow{2}{*}{$\begin{array}{l}\mathbf{N}_{2}^{\mathrm{g}} \\
(\%)\end{array}$} & \multirow{2}{*}{$\begin{array}{c}\mathbf{C H}_{4}^{\mathrm{g}} \\
(\%)\end{array}$} & \multirow{2}{*}{$\begin{array}{c}\mathrm{CO}_{2}^{\mathrm{g}} \\
(\%)\end{array}$} & \multirow{2}{*}{$\begin{array}{c}\text { Ebullitive } \\
\mathrm{N}_{2} \mathrm{aec}-\mathrm{N} \\
\left(\mathrm{mg} \cdot \mathrm{m}^{-2} \cdot \mathrm{d}^{-1}\right)\end{array}$} \\
\hline $\begin{array}{l}\text { Latitude } \\
\left({ }^{\circ} \mathrm{S}\right)\end{array}$ & $\begin{array}{l}\text { Longitude } \\
\left({ }^{\circ} \mathrm{W}\right)\end{array}$ & & & & & & & & & & & \\
\hline 3.3118 & 52.1960 & 3.5 & 2000 & 4 & 22.18 & 301.7 & 747.0 & 4.11 & 13.0 & 84.4 & 0.46 & $0(-66.5)^{\dagger}$ \\
\hline 3.3118 & 52.1960 & 4.5 & 583 & 3 & 22.72 & 301.7 & 747.0 & 6.40 & 25.1 & 68.3 & 0.14 & $0(-10.5)$ \\
\hline 3.3118 & 52.1960 & 5.5 & 520 & 4 & 22.33 & 301.7 & 747.0 & 4.89 & 26.5 & 68.3 & 0.21 & $24.1^{\mathrm{h}}$ \\
\hline 3.4145 & 52.2497 & 3 & 320 & 4 & 22.72 & 301.6 & 746.0 & 5.49 & 19.4 & 75.0 & 0.19 & $0(-8.90)$ \\
\hline 3.4145 & 52.2497 & 4.5 & $<1.6$ & 3 & 22.75 & 301.6 & 746.0 & $\mathrm{na}^{\mathrm{m}}$ & na & na & na & 0 \\
\hline 3.4145 & 52.2497 & 5.5 & 957 & 4 & 21.95 & 301.6 & 746.0 & 22.6 & 77.1 & 0.20 & 0.13 & $0(-134)$ \\
\hline 3.3668 & 51.9870 & 4.5 & $<1.6$ & 4 & 20.47 & 301.5 & 748.0 & na & na & na & na & 0 \\
\hline 3.3668 & 51.9870 & 3.5 & 1464 & 4 & 20.35 & 301.5 & 748.0 & 4.31 & 24.5 & 71.2 & 0.57 & 80.0 \\
\hline 3.3668 & 51.9870 & 4.5 & 29 & 3 & 19.83 & 301.5 & 748.0 & 8.96 & 43.4 & 49.4 & 0.18 & 1.96 \\
\hline 3.2159 & 52.1114 & 3.5 & 135 & 4 & 22.50 & 301.8 & 761.0 & 6.34 & 36.5 & 56.3 & 0.23 & 10.5 \\
\hline 3.2159 & 52.1114 & 4.5 & 20 & 4 & 21.92 & 301.8 & 761.0 & 11.8 & 60.8 & 26.9 & 0.17 & 1.81 \\
\hline 3.2159 & 52.1114 & 4 & $<1.6$ & 3 & 21.93 & 301.8 & 761.0 & na & na & na & na & 0 \\
\hline 3.2129 & 52.1874 & 4 & 38 & 3 & 22.63 & 302.8 & 745.0 & 12.3 & 65.3 & 23.9 & 0.46 & 5.26 \\
\hline 3.2129 & 52.1874 & 2.5 & 10 & 2 & 22.92 & 302.8 & 745.0 & 2.68 & 79.0 & 17.9 & 0.42 & 10.2 \\
\hline 3.2020 & 52.1946 & 4 & 14 & 4 & 23.20 & 301.0 & 745.0 & 14.2 & 83.2 & 1.36 & 0.22 & 2.46 \\
\hline 3.2020 & 52.1946 & 2 & $<1.6$ & 2 & 23.25 & 301.0 & 745.0 & na & na & na & na & 0 \\
\hline \multicolumn{12}{|c|}{ Xingu River (1/2) May 13 to 18,2008 survey $\mathrm{N}_{2}$ aec bubble-emission median $(\mathrm{n}=16)$} & $0.91(0.91)$ \\
\hline 3.4032 & 52.2597 & 1.5 & $<1.6$ & 2 & 23.07 & 304.0 & 746.0 & na & na & na & na & 0 \\
\hline 3.4032 & 52.2597 & 2 & $<1.6$ & 3 & 23.72 & 304.0 & 746.0 & na & na & na & na & 0 \\
\hline 3.4032 & 52.2597 & 3 & 8 & 3 & 23.90 & 304.0 & 746.0 & 21.5 & 78.3 & 0.17 & 0.04 & $0(-0.92)$ \\
\hline 3.3402 & 52.1960 & 1.5 & 4 & 3 & 24.33 & 304.5 & 746.0 & 18.5 & 81.5 & na & na & 0.14 \\
\hline 3.3402 & 52.1960 & 3 & 11 & 2 & 24.20 & 304.5 & 746.0 & 19.8 & 80.1 & 0.01 & 0.06 & $0(-0.47)$ \\
\hline 3.3402 & 52.1960 & 4 & $<1.6$ & 2 & 24.23 & 304.5 & 746.0 & na & na & na & na & 0 \\
\hline 3.2183 & 52.1119 & 1.5 & $<1.6$ & 2 & 17.55 & 306.0 & 747.0 & na & na & na & na & 0 \\
\hline 3.2183 & 52.1119 & 2 & 5 & 2 & 17.42 & 306.0 & 747.0 & 20.9 & 81.7 & 0.03 & 0.05 & $0(-0.59)$ \\
\hline 3.2183 & 52.1119 & 3 & $<1.6$ & 2 & 17.12 & 306.0 & 747.0 & na & na & na & na & 0 \\
\hline 3.2157 & 52.1741 & 1.5 & 6 & 3 & 16.28 & 306.4 & 747.0 & 19.8 & 80.3 & 0.87 & 0.05 & $0(-0.24)$ \\
\hline 3.2157 & 52.1918 & 2.5 & $<1.6$ & 2 & 15.82 & 305.3 & 747.0 & na & na & na & na & 0 \\
\hline 3.2157 & 52.1918 & 5 & 6 & 2 & 15.55 & 305.3 & 747.0 & 21.7 & 78.0 & 0.02 & 0.09 & $0(-1.73)$ \\
\hline 3.3729 & 51.9495 & 1.5 & 39 & 3 & 22.05 & 304.8 & 745.0 & 10.2 & 61.2 & 28.5 & 0.12 & 7.41 \\
\hline 3.3729 & 51.9495 & 2.5 & 3 & 2 & 22.22 & 304.8 & 745.0 & 15.6 & 78.0 & 6.32 & 0.08 & 0.58 \\
\hline 3.3729 & 51.9495 & 3.5 & $<1.6$ & 3 & 22.10 & 304.8 & 745.0 & na & na & na & na & 0 \\
\hline 3.3681 & 51.9773 & 1.5 & 8 & 4 & 21.03 & 305.4 & 745.0 & 21.8 & 78.1 & 0.08 & 0.05 & $0(-0.87)$ \\
\hline 3.3681 & 51.9773 & 4 & $<1.6$ & 3 & 20.62 & 305.4 & 745.0 & na & na & na & na & 0 \\
\hline 3.2491 & 52.0549 & 1.5 & 23 & 3 & 18.08 & 304.9 & 746.0 & 21.5 & 78.4 & 0.03 & 0.09 & $0(-3.45)$ \\
\hline 3.2491 & 52.0549 & 2 & $<1.6$ & 2 & 18.30 & 304.9 & 746.0 & na & na & na & na & 0 \\
\hline 3.2491 & 52.0549 & 3.5 & 11 & 2 & 17.90 & 304.9 & 746.0 & 19.3 & 80.6 & 0.03 & 0.10 & $0(-0.09)$ \\
\hline 3.2453 & 52.0639 & 1.5 & 31 & 4 & 17.92 & 305.1 & 746.0 & 6.79 & 64.2 & 28.6 & 0.37 & 10.6 \\
\hline 3.2453 & 52.0639 & 2.5 & $<1.6$ & 3 & 18.22 & 305.1 & 746.0 & na & na & na & na & 0 \\
\hline \multicolumn{12}{|c|}{ Xingu River $(2 / 2)$ Oct 16 to 21,2008 survey $\mathrm{N}_{2}$ aec bubble-emission median $(\mathrm{n}=22)$} & $0(0)$ \\
\hline \multicolumn{12}{|c|}{$\begin{array}{l}\text { Xingu River } \mathrm{N}_{2} \text { aec bubble-emission median }(\mathrm{n}=38) \\
\text { (first quartile; third quartile) }\end{array}$} & $\begin{array}{c}0(0 ; 1.50) \\
0(-0.41 ; 1.50)\end{array}$ \\
\hline
\end{tabular}


(b)

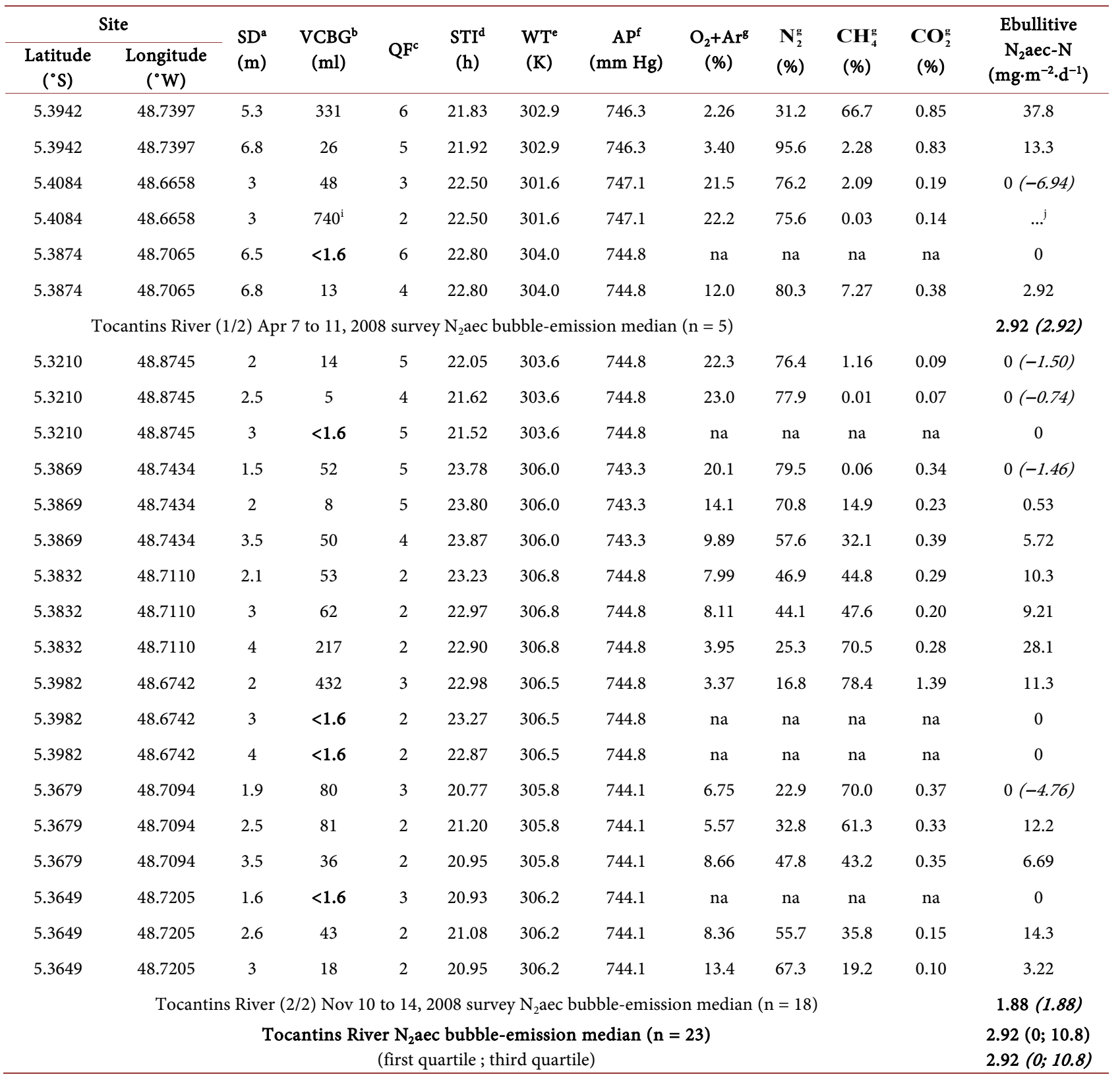

(c)

\begin{tabular}{|c|c|c|c|c|c|c|c|c|c|c|c|c|}
\hline \multicolumn{2}{|c|}{ Site } & \multirow{2}{*}{$\begin{array}{l}S^{a} \\
(m)\end{array}$} & \multirow{2}{*}{$\begin{array}{c}\mathrm{VCBG}^{\mathrm{b}} \\
(\mathrm{ml})\end{array}$} & \multirow{2}{*}{$\mathrm{QF}^{\mathrm{c}}$} & \multirow{2}{*}{$\begin{array}{l}\mathrm{STI}^{\mathrm{d}} \\
(\mathrm{h})\end{array}$} & \multirow{2}{*}{$\begin{array}{l}\mathrm{WT}^{\mathrm{e}} \\
(\mathrm{K})\end{array}$} & \multirow{2}{*}{$\begin{array}{c}\mathrm{AP}^{\mathrm{f}} \\
(\mathrm{mm} \cdot \mathrm{Hg})\end{array}$} & \multirow{2}{*}{$\begin{array}{c}\mathrm{O}_{2}+\mathrm{Ar}^{\mathrm{g}} \\
(\%)\end{array}$} & \multirow{2}{*}{$\begin{array}{l}N_{2}^{g} \\
(\%)\end{array}$} & \multirow{2}{*}{$\begin{array}{c}\mathrm{CH}_{4}^{\mathrm{g}} \\
(\%)\end{array}$} & \multirow{2}{*}{$\begin{array}{c}\mathrm{CO}_{2}^{\mathrm{g}} \\
(\%)\end{array}$} & \multirow{2}{*}{$\begin{array}{c}\text { Ebullitive } \\
\mathrm{N}_{2} \text { aec-N } \\
\left(\mathrm{mg} \cdot \mathrm{m}^{-2} \cdot \mathrm{d}^{-1}\right)\end{array}$} \\
\hline $\begin{array}{c}\text { Latitude } \\
\left({ }^{\circ} \mathrm{S}\right)\end{array}$ & $\begin{array}{c}\text { Longitude } \\
\left({ }^{\circ} \mathrm{W}\right)\end{array}$ & & & & & & & & & & & \\
\hline 8.8516 & 64.0644 & 3 & 790 & 2 & 20.50 & 301.1 & 750.8 & 9.69 & 42.4 & 46.7 & 1.23 & 23.0 \\
\hline 8.8621 & 64.0593 & 3 & 1370 & 3 & 23.10 & 300.6 & 750.8 & 17.4 & 80.3 & 1.13 & 0.58 & 100 \\
\hline 8.8584 & 64.0241 & 4.7 & 1430 & 2 & 22.62 & 300.8 & 750.8 & 16.5 & 74.2 & 8.57 & 0.69 & 109 \\
\hline 8.9153 & 64.0899 & 4 & 620 & 3 & 19.25 & 298.4 & 748.6 & 4.86 & 28.8 & 65.1 & 1.25 & 63.3 \\
\hline 8.9855 & 64.1237 & 3.9 & 310 & 1 & 19.25 & 302.5 & 748.6 & 5.59 & 65.1 & 28.3 & 0.96 & 464 \\
\hline 8.9983 & 64.1323 & 5.6 & 210 & 3 & 19.33 & 302.5 & 748.6 & 6.89 & 41.8 & 50.7 & 0.61 & 32.3 \\
\hline 9.0368 & 64.1954 & 2.2 & 350 & 3 & 20.33 & 300.8 & 747.8 & 5.38 & 34.3 & 58.2 & 2.10 & 46.8 \\
\hline
\end{tabular}




\section{Continued}

\begin{tabular}{|c|c|c|c|c|c|c|c|c|c|c|c|c|}
\hline 9.1160 & 64.3176 & 4 & 230 & 4 & 19.92 & 300.5 & 747.8 & 4.4 & 51.1 & 43.7 & 0.83 & 65.6 \\
\hline 9.2094 & 64.3897 & 5 & 500 & 1 & 19.42 & 300.9 & 747.8 & 3.46 & 18.7 & 74.9 & 2.99 & 74.7 \\
\hline 9.1818 & 64.5136 & 6 & 78 & 1 & 19.55 & $300.9^{\mathrm{k}}$ & 748.6 & 22.2 & 77.5 & 0.06 & 0.06 & $0(-43.7)$ \\
\hline 9.2458 & 64.6233 & 3 & 110 & 3 & 24.00 & $300.9^{k}$ & 748.6 & 22.2 & 78.4 & 0.41 & 0.06 & $0(-15.8)$ \\
\hline 8.8390 & 64.0155 & 9.6 & 365 & 2 & 20.28 & 301.1 & 750.8 & 15.5 & 77.9 & 6.07 & 0.50 & 80.5 \\
\hline 8.9883 & 64.1015 & 3.5 & 25 & 2 & 19.25 & 302.8 & 748.6 & 15.9 & 71.0 & 12.7 & 0.45 & 1.90 \\
\hline \multicolumn{12}{|c|}{ Madeira River (1/2) May 27 to 30, 2011 survey $\mathrm{N}_{2}$ aec bubble-emission median $(\mathrm{n}=13)$} & $63.3(63.3)$ \\
\hline 8.8396 & 64.0159 & 9.9 & 30 & 2 & 23.92 & 299.7 & 750.1 & 5.9 & 38.0 & 55.6 & 0.56 & 5.81 \\
\hline 8.8527 & 64.0647 & 7.1 & 280 & 3 & 23.95 & 301.4 & 750.1 & 11.9 & 70.0 & 17.7 & 0.46 & 54.4 \\
\hline 8.8343 & 63.9674 & 4.8 & 90 & 1 & 24.03 & 300.3 & 750.1 & 6.1 & 31.2 & 62.1 & 0.63 & 14.6 \\
\hline 8.7950 & 63.9716 & 11.0 & 210 & 2 & 23.93 & 299.9 & 750.1 & 8.5 & 67.3 & 23.9 & 0.44 & 97.1 \\
\hline 9.0417 & 64.2963 & 1.2 & 160 & 2 & 21.13 & 300.7 & 749.3 & 3.87 & 45.3 & 50.2 & 0.63 & 76.7 \\
\hline 9.1083 & 64.3161 & 5.5 & 380 & 2 & 22.30 & 300.1 & 749.3 & 7.87 & 38.8 & 52.8 & 0.46 & 34.3 \\
\hline 8.9748 & 64.1008 & 6.0 & 500 & 1 & 23.50 & 299.9 & 749.3 & 4.42 & 20.3 & 74.8 & 0.48 & 25.9 \\
\hline 9.0145 & 64.1675 & 4.3 & $300^{1}$ & 3 & 20.25 & 300.4 & 749.3 & 2.17 & 17.4 & 79.3 & 1.21 & 28.6 \\
\hline 9.2101 & 64.4191 & 4.9 & $500^{1}$ & 2 & 22.68 & 300.0 & 750.1 & 2.13 & 20.5 & 75.5 & 1.83 & 89.1 \\
\hline 9.1438 & 64.5152 & 2.3 & 420 & 2 & 21.43 & 299.8 & 750.1 & 18.5 & 70.6 & 10.5 & 0.43 & $O(-48.6)$ \\
\hline 9.1827 & 64.6129 & 7.0 & 60 & 2 & 20.37 & 299.7 & 750.1 & 7.89 & 51.7 & 40.0 & 0.42 & 19.2 \\
\hline 9.2582 & 64.6313 & 3.0 & 10 & 2 & 19.13 & 299.7 & 750.1 & 13.2 & 82.5 & 3.91 & 0.43 & 4.96 \\
\hline 9.2226 & 64.6192 & 2.0 & $1000^{1}$ & 2 & 19.13 & 299.9 & 750.1 & 1.8 & 13.3 & 82.3 & 2.49 & 105 \\
\hline \multicolumn{12}{|c|}{ Madeira River (2/2) March 7 to 9, 2012 survey $\mathrm{N}_{2}$ aec bubble-emission median $(\mathrm{n}=13)$} & $28.6(28.6)$ \\
\hline & & & ira Riv & $\mathrm{N}_{2} \mathrm{ae}$ & ubble- & ission $\mathrm{n}$ & $\operatorname{ian}(n=$ & & & & & $40.5(15.8 ; 79.6)$ \\
\hline & & & & rst c & rtile ; tl & d quartil & & & & & & $40.5(15.8 ; 79.6)$ \\
\hline
\end{tabular}

(d)

\begin{tabular}{|c|c|c|c|c|c|c|c|c|c|c|c|c|}
\hline \multicolumn{2}{|c|}{ Site } & \multirow{2}{*}{$\begin{array}{l}\mathrm{SD}^{\mathrm{a}} \\
(\mathrm{m})\end{array}$} & \multirow{2}{*}{$\begin{array}{l}\mathrm{VCBG}^{\mathrm{b}} \\
(\mathrm{ml})\end{array}$} & \multirow[b]{2}{*}{$\mathrm{QF}^{\mathrm{c}}$} & \multirow{2}{*}{$\begin{array}{c}\mathrm{STI}^{\mathrm{d}} \\
(\mathrm{h})\end{array}$} & \multirow{2}{*}{$\begin{array}{l}\mathrm{WT}^{\mathrm{e}} \\
(\mathrm{K})\end{array}$} & \multirow{2}{*}{$\begin{array}{c}\mathrm{AP}^{\mathrm{f}} \\
(\mathrm{mm} \cdot \mathrm{Hg})\end{array}$} & \multirow{2}{*}{$\begin{array}{c}\mathrm{O}_{2}+\mathrm{Ar}^{\mathrm{g}} \\
(\%)\end{array}$} & \multirow{2}{*}{$\begin{array}{l}\mathbf{N}_{2}^{\mathrm{g}} \\
(\%)\end{array}$} & \multirow{2}{*}{$\begin{array}{c}\mathrm{CH}_{4}^{\mathrm{g}} \\
(\%)\end{array}$} & \multirow{2}{*}{$\begin{array}{c}\mathrm{CO}_{2}^{\mathrm{g}} \\
(\%)\end{array}$} & \multirow{2}{*}{$\begin{array}{c}\text { Ebullitive } \\
\mathrm{N}_{2} \text { aec-N } \\
\left(\mathrm{mg} \cdot \mathrm{m}^{-2} \cdot \mathrm{d}^{-1}\right)\end{array}$} \\
\hline $\begin{array}{c}\text { Latitude } \\
\left({ }^{\circ} \mathrm{S}\right)\end{array}$ & $\begin{array}{l}\text { Longitude } \\
\left({ }^{\circ} \mathrm{W}\right)\end{array}$ & & & & & & & & & & & \\
\hline 17.0235 & 47.1476 & 4.2 & 275 & 5 & 21.28 & 297.3 & 699.1 & 20.9 & 77.4 & 1.38 & 0.32 & $0(-17.6)$ \\
\hline 17.0618 & 47.1892 & 5.9 & 80 & 4 & 14.85 & 296.9 & 697.6 & 15.4 & 59.4 & 15.4 & 0.34 & $0(-4.66)$ \\
\hline 17.0600 & 47.1894 & 4.0 & 280 & 7 & 14.28 & 297.1 & 697.6 & 9.02 & 32.4 & 57.8 & 0.78 & $0(-10.1)$ \\
\hline 17.0991 & 47.2483 & 6 & 410 & 6 & 16.73 & 297.3 & 697.6 & 4.35 & 31.0 & 64.3 & 0.38 & 34.2 \\
\hline 17.1000 & 47.2653 & 3 & 1060 & 4 & 18.10 & 296.0 & 697.6 & 4.86 & 30.6 & 63.6 & 0.97 & 98.4 \\
\hline 16.9594 & 47.1598 & 2 & 1199 & 7 & 21.53 & 297.3 & 699.1 & 7.55 & 77.8 & 14.3 & 0.30 & 241 \\
\hline 16.9594 & 47.1599 & 4 & 1075 & 6 & 21.85 & 297.1 & 699.1 & 4.74 & 33.1 & 61.3 & 0.81 & 71.3 \\
\hline 16.9641 & 47.1550 & 4.5 & 2375 & 6 & 21.95 & 297.4 & 699.1 & 3.07 & 15.9 & 80.4 & 0.64 & 35.7 \\
\hline 17.1418 & 47.2958 & 5 & 340 & 5 & 21.58 & 297.2 & 698.3 & 4.54 & 23.2 & 72.0 & 0.30 & 8.57 \\
\hline 17.2092 & 47.3629 & 4.8 & 30 & 5 & 20.27 & 297.1 & 698.3 & 17.7 & 65.6 & 16.5 & 0.24 & $0(-1.70)$ \\
\hline 17.2047 & 47.3602 & 4.6 & 760 & 5 & 19.32 & 297.1 & 698.3 & 7.17 & 26.1 & 66.4 & 0.35 & $0(-20.7)$ \\
\hline 17.2271 & 47.3609 & 1.5 & 100 & 6 & 22.17 & 295.8 & 698.3 & 4.95 & 27.6 & 65.8 & 1.64 & 3.39 \\
\hline 17.2229 & 47.3650 & 6 & 2000 & 4 & 21.20 & 295.7 & 698.3 & 4.01 & 13.5 & 81.7 & 0.76 & $0(-52.0)$ \\
\hline 17.2369 & 47.4148 & 3 & 65 & 7 & 20.92 & 297.1 & 697.6 & 12.9 & 59.2 & 27.4 & 0.52 & 1.47 \\
\hline
\end{tabular}


E. Sikar et al.

Continued

\begin{tabular}{|c|c|c|c|c|c|c|c|c|c|c|c|c|}
\hline 17.2125 & 47.4170 & 3 & 30 & 3 & 20.70 & 296.5 & 697.6 & 18.2 & 69.9 & 10.9 & 0.17 & $0(-2.07)$ \\
\hline 17.3114 & 47.4242 & 6.6 & 575 & 6 & 20.85 & 296.7 & 697.6 & 4.38 & 27.9 & 67.3 & 0.47 & 28.8 \\
\hline 17.2769 & 47.5227 & 4.5 & 153 & 3 & 21.30 & 297.1 & 697.6 & 3.75 & 21.0 & 74.8 & 0.48 & 8.27 \\
\hline 17.3470 & 47.4844 & 5.2 & 510 & 3 & 21.33 & 297.1 & 697.6 & 21.2 & 78.4 & 0.24 & 0.19 & $0(-55.6)$ \\
\hline \multicolumn{12}{|c|}{ São Marcos River \& tributaries (1/3) Mar 21 to 25, 2011 survey $\mathrm{N}_{2}$ aec bubble-emission median $(\mathrm{n}=18)$} & $2.43(2.43)$ \\
\hline 17.0269 & 47.1462 & 2 & 480 & 2 & 20.28 & 292.6 & 702.1 & 17.8 & 68.5 & 13.6 & 0.10 & $0(-49.6)$ \\
\hline 17.0382 & 47.1791 & 1 & 190 & 3 & 19.13 & 293.2 & 702.1 & 15.8 & 81.6 & 1.86 & 0.14 & 33.9 \\
\hline 17.0626 & 47.1902 & 3.9 & 260 & 3 & 20.42 & 292.7 & 702.1 & 6.79 & 51.4 & 41.6 & 0.27 & 65.2 \\
\hline 17.1000 & 47.2492 & 4.6 & 130 & 3 & 19.23 & 292.6 & 702.1 & 6.77 & 46.7 & 46.4 & 0.18 & 27.7 \\
\hline 16.9646 & 47.1546 & 2.6 & 70 & 3 & 20.75 & 292.3 & 702.1 & 19.4 & 76.0 & 4.44 & 0.11 & $0(-4.13)$ \\
\hline 17.1461 & 47.3026 & 3 & 10 & 3 & 21.03 & 292.2 & 700.6 & 19.9 & 78.4 & 1.60 & 0.10 & $0(-0.55)$ \\
\hline 17.2068 & 47.3618 & 4.3 & 10 & 3 & 21.15 & 292.5 & 700.6 & 20.4 & 79.5 & na & 0.07 & $0(-0.65)$ \\
\hline 17.2106 & 47.3623 & 5 & 45 & 3 & 21.15 & 292.6 & 700.6 & 20.5 & 79.2 & 0.04 & 0.12 & $0(-3.27)$ \\
\hline 17.2428 & 47.3960 & 1.8 & 80 & 3 & 21.00 & 292.8 & 700.6 & 20.8 & 78.6 & 2.22 & 0.10 & $0(-7.43)$ \\
\hline 17.3128 & 47.4241 & 2.5 & 1000 & 2 & 21.87 & 292.1 & 701.3 & 2.82 & 9.66 & 80.3 & 7.25 & $0(-33.5)$ \\
\hline 17.3486 & 47.4869 & 4.8 & 180 & 3 & 21.30 & 292.3 & 701.3 & 16.6 & 59.6 & 23.2 & 0.51 & $0(-19.1)$ \\
\hline \multicolumn{12}{|c|}{ São Marcos River \& tributaries (2/3) June 13 to 17,2011 survey $\mathrm{N}_{2}$ aec bubble-emission median $(\mathrm{n}=11)$} & $0(-3.27)$ \\
\hline 17.0258 & 47.1488 & 1.8 & 35 & 2 & 23.07 & 295.5 & 696.8 & 17.1 & 76.1 & 6.56 & 0.23 & 2.15 \\
\hline 17.0620 & 47.1895 & 1.9 & 30 & 3 & 22.90 & 296.7 & 696.8 & 3.61 & 23.8 & 71.5 & 1.12 & 2.48 \\
\hline 17.0357 & 47.1798 & 2 & $\ldots$ & 3 & 22.82 & 295.9 & 696.8 & 3.65 & 39.8 & 56.1 & 0.46 & $\ldots$ \\
\hline 17.1000 & 47.2492 & 4.1 & 10 & 3 & 22.75 & 297.5 & 696.8 & 5.53 & 50.3 & 43.8 & 0.31 & 2.60 \\
\hline 17.1188 & 47.2798 & 2 & 20 & 3 & 24.42 & 296.0 & 696.8 & 19.5 & 80.2 & 0.05 & 0.30 & $0(-0.30)$ \\
\hline 17.1482 & 47.3272 & 2.3 & 240 & 3 & 24.48 & 296.5 & 696.8 & 19.2 & 80.5 & 0.04 & 0.25 & $0(-0.26)$ \\
\hline 17.2224 & 47.3629 & 3.2 & 60 & 2 & 27.35 & 297.4 & 698.3 & 4.52 & 27.5 & 66.3 & 1.62 & 6.14 \\
\hline 17.2449 & 47.4044 & 1.2 & 30 & 3 & 18.83 & 296.3 & 698.3 & 14.9 & 64.1 & 20.8 & 0.26 & 0.54 \\
\hline 17.3071 & 47.4276 & 2 & 200 & 3 & 22.98 & 297.1 & 698.3 & 18.1 & 81.1 & 0.55 & 0.24 & 9.73 \\
\hline \multicolumn{12}{|c|}{ São Marcos River \& tributaries (3/3) Sept 26 to 30, 2011 survey $\mathrm{N}_{2}$ aec bubble-emission median $(\mathrm{n}=8)$} & $2.32(2.32)$ \\
\hline \multicolumn{12}{|c|}{ São Marcos River and tributaries $\mathrm{N}_{2}$ aec bubble-emission median $(\mathrm{n}=37)$} & $0.54(0 ; 9.73)$ \\
\hline \multicolumn{12}{|c|}{ (first quartile; third quartile) } & $0.54(-4.66 ; 9.73)$ \\
\hline
\end{tabular}

(e)

\begin{tabular}{|c|c|c|c|c|c|c|c|c|c|c|c|c|}
\hline \multicolumn{2}{|c|}{ Site } & \multirow[b]{2}{*}{$\mathrm{SD}^{\mathrm{a}}(\mathrm{m})$} & \multirow{2}{*}{$\begin{array}{c}\mathrm{VCBG}^{\mathrm{b}} \\
(\mathrm{ml})\end{array}$} & \multirow[b]{2}{*}{$\mathrm{QF}^{\mathrm{c}}$} & \multirow[b]{2}{*}{$\begin{array}{c}\mathrm{STI}^{\mathrm{d}} \\
(\mathrm{h})\end{array}$} & \multirow{2}{*}{$\begin{array}{l}\mathrm{WT}^{\mathrm{e}} \\
(\mathrm{K})\end{array}$} & \multirow{2}{*}{$\begin{array}{c}\mathrm{AP}^{\mathrm{f}} \\
(\mathrm{mm} \mathrm{Hg})\end{array}$} & \multirow[b]{2}{*}{$\begin{array}{c}\mathrm{O}_{2}+ \\
\operatorname{Ar}^{\mathrm{g}}(\%)\end{array}$} & \multirow[b]{2}{*}{$\begin{array}{l}\mathbf{N}_{2}^{\mathrm{g}} \\
(\%)\end{array}$} & \multirow[b]{2}{*}{$\begin{array}{c}\mathrm{CH}_{4}^{\mathrm{g}} \\
(\%)\end{array}$} & \multirow[b]{2}{*}{$\begin{array}{c}\mathrm{CO}_{2}^{\mathrm{g}} \\
(\%)\end{array}$} & \multirow{2}{*}{$\begin{array}{c}\text { Ebullitive } \\
\mathrm{N}_{2} \mathrm{aec}-\mathrm{N} \\
\left(\mathrm{mg} \cdot \mathrm{m}^{-2} \cdot \mathrm{d}^{-1}\right)\end{array}$} \\
\hline $\begin{array}{c}\text { Latitude } \\
\left({ }^{\circ} \mathrm{S}\right)\end{array}$ & $\begin{array}{c}\text { Longitude } \\
\left({ }^{\circ} \mathrm{W}\right)\end{array}$ & & & & & & & & & & & \\
\hline 23.5254 & 46.7501 & 1.7 & 564 & 3 & 2.33 & 294.2 & 699.8 & 1.02 & 10.9 & 84.2 & 3.92 & 355 \\
\hline 23.5067 & 46.5474 & 2.3 & 855 & 2 & 9.00 & 294.2 & 699.8 & 1.65 & 14.2 & 80.8 & 3.41 & 230 \\
\hline 23.5255 & 46.7501 & 1.7 & 5400 & 3 & 18.75 & 294.2 & 696.1 & 1.58 & 13.8 & 79.7 & 4.95 & 456 \\
\hline
\end{tabular}

Tietê River (1/1) May 8 to 9, 2012 survey $\mathrm{N}_{2}$ aec bubble-emission median $(\mathrm{n}=3)$ (first quartile; third quartile)

$355(293 ; 405)^{\mathrm{n}}$

(f)

\begin{tabular}{|c|c|c|c|c|c|c|c|c|c|c|c|c|}
\hline \multicolumn{2}{|c|}{ Site } & \multirow{2}{*}{$\begin{array}{l}\mathrm{SD}^{\mathrm{a}} \\
(\mathrm{m})\end{array}$} & \multirow{2}{*}{$\begin{array}{c}\mathrm{VCBG}^{\mathrm{b}} \\
(\mathrm{ml})\end{array}$} & \multirow[b]{2}{*}{$\mathrm{QF}^{\mathrm{c}}$} & \multirow{2}{*}{$\begin{array}{l}\text { STI }^{d} \\
(\mathrm{~h})\end{array}$} & \multirow{2}{*}{$\begin{array}{l}\mathrm{WT}^{\mathrm{e}} \\
(\mathrm{K})\end{array}$} & \multirow{2}{*}{$\begin{array}{c}\mathrm{AP}^{\mathrm{f}} \\
(\mathrm{mm} \mathrm{Hg})\end{array}$} & \multirow{2}{*}{$\begin{array}{c}\mathrm{O}_{2}+\mathrm{Ar}^{\mathrm{g}} \\
(\%)\end{array}$} & \multirow{2}{*}{$\begin{array}{l}\mathrm{N}_{2}^{\mathrm{g}} \\
(\%)\end{array}$} & \multirow{2}{*}{$\begin{array}{c}\mathrm{CH}_{4}^{\mathrm{g}} \\
(\%)\end{array}$} & \multirow{2}{*}{$\begin{array}{l}\mathrm{CO}_{2}^{\mathrm{g}} \\
(\%)\end{array}$} & \multirow{2}{*}{$\begin{array}{c}\text { Ebullitive } \\
\mathrm{N}_{2} \mathrm{aec}-\mathrm{N} \\
\left(\mathrm{mg} \cdot \mathrm{m}^{-2} \cdot \mathrm{d}^{-1}\right)\end{array}$} \\
\hline $\begin{array}{c}\text { Latitude } \\
\left({ }^{\circ} \mathrm{S}\right)\end{array}$ & $\begin{array}{c}\text { Longitude } \\
\left({ }^{\circ} \mathrm{W}\right)\end{array}$ & & & & & & & & & & & \\
\hline 23.5255 & 46.7494 & 3 & 4200 & 3 & 0.85 & 293.9 & 699.8 & 1.10 & 5.61 & 80.7 & 12.6 & 1088 \\
\hline 23.5332 & 46.7488 & 1.9 & 305 & 3 & 1.08 & 293.9 & 696.1 & 1.02 & 16.8 & 80.4 & 1.80 & 781 \\
\hline
\end{tabular}

Pinheiros River (1/1) May 8 to 9, 2012 survey $\mathrm{N}_{2}$ aec bubble-emission median $(\mathrm{n}=2)$ 